\title{
A computational model of drug delivery through microcirculation to compare different tumor treatments
}

\author{
L. Cattaneo ${ }^{1}$ and P. Zunino ${ }^{2, *, \dagger}$ \\ ${ }^{1}$ MOX, Department of Mathematics “Francesco Brioschi”, Politecnico di Milano, Piazza Leonardo da Vinci 32, \\ 20133 Milano, Italy \\ ${ }^{2}$ Department of Mechanical Engineering and Materials Science, University of Pittsburgh, 3700 O'Hara Street, \\ Pittsburgh, PA 15261, U.S.A.
}

\begin{abstract}
SUMMARY
Starting from the fundamental laws of filtration and transport in biological tissues, we develop a computational model to capture the interplay between blood perfusion, fluid exchange with the interstitial volume, mass transport in the capillary bed, through the capillary walls and into the surrounding tissue. These phenomena are accounted at the microscale level, where capillaries and interstitial volume are viewed as two separate regions. The capillaries are described as a network of vessels carrying blood flow. We apply the model to study drug delivery to tumors. The model can be adapted to compare various treatment options. In particular, we consider delivery using drug bolus injection and nanoparticle injection into the blood stream. The computational approach is suitable for a systematic quantification of the treatment performance, enabling the analysis of interstitial drug concentration levels, metabolization rates and cell surviving frac-tions. Our study suggests that for the treatment based on bolus injection, the drug dose is not optimally delivered to the tumor interstitial volume. Using nanoparticles as intermediate drug carriers overrides the shortcomings of the previous delivery approach. This work shows that the proposed theoretical and com-putational framework represents a promising tool to compare the efficacy of different cancer treatments.
\end{abstract}

Received 1 February 2014; Revised 6 May 2014; Accepted 30 June 2014

KEY WORDS: blood perfusion; mass transport; microvascular environment; drug delivery

\section{INTRODUCTION}

Mass transport plays a fundamental role in the development of cancer. At different phases of cancer disease, such as the propagation of growth signals, the invasion of other tissue and the activation of angiogenesis, tumors use mass transport phenomena to interact with the surrounding environment [1]. Mass transport is also at the basis of cancer pharmacological treatment. Targeting vascularized tumors using the vascular network is a natural therapeutic option. Nevertheless, the success of anticancer therapies in treating cancer cells is limited by their inability to reach their target in vivo in adequate quantities [2]. An agent that is delivered intravenously reaches cancer cells via distribution through the vasculature, transport across the wall of the vessels and transport through the tissue interstitium. Each of these steps can be seen as a barrier to delivery. In addition, delivered molecules may bind to constituents of the extracellular matrix and be metabolized by cells.

The characteristic traits of cancer can be seen as the emergent effects of a cascade of phenomena that propagate from the molecular scale, through the cell and the tissue microenvironment, up to the systemic level. Transport phenomena at the level of the capillary network (the microenvironment or microscale) play a key role in this sequence of effects. In particular, the alterations of the

*Correspondence to: P. Zunino, Department of Mechanical Engineering and Materials Science, University of Pittsburgh, 3700 O'Hara Street, Pittsburgh, PA 15261, U.S.A.

†E-mail: paz13@pitt.edu 
capillary phenotype of a tumor significantly affect the drug delivery process [3]. More precisely, blood vessels in tumors are leakier and more tortuous than the normal vasculature and the pressure generated by the proliferating cells reduces tumor blood and lymphatic flow. These perturbations lead to an impaired blood supply and abnormal tumor microenvironment characterized by hypoxia and elevated interstitial fluid pressure. They also reduce the ability to deliver drugs.

The objective of this work is to perform a comparative study of different modalities to deliver drug to a vascularized tumor mass. This is achieved by developing a new computational pharmacokinetic model able to capture the absorption of a drug through the vascular network as well as its distribution and metabolization in the tumor. Following the seminal sequence of works by Baxter and Jain [4-7], we believe that the interplay between blood perfusion, fluid exchange with the interstitial volume, mass transport in the capillary bed, through the capillary walls and into the surrounding tissue, are important effects to understand the delivery process at the microscale. Temporal and spatial dependence will be fully accounted in our governing equations, in contrast to the approach based on compartment models. Because we consider these phenomena at the level of capillaries, it is possible to derive the governing equations from a mechanistic standpoint based on the fundamental laws of flow and mass transport. The model is also suitable for being adapted to different delivery methods. Besides studying the case of bolus injection, which consists in delivering a solution containing the active drug into the peripheral systemic circulation, we analyze the delivery of drug from nanoparticles, which are in turn injected into the blood stream and interact with the capillary walls.

The analysis of tissue perfusion and mass transport has been addressed using various advanced approximation approaches. Without any ambition to provide an exhaustive literature review, we mention $[8,9]$ where the problem of cardiac perfusion is addressed, $[10,11]$ where homogenization techniques are applied to characterize the average transport properties of tumor tissue constructs and [12] where isogeometric analysis is used to model angiogenesis in vascularized tumors.

The model that we develop ends up to be a system of partial differential equations, which are hard to solve with analytical tools. For this reason, we complement the model with a state of art numerical solver, based on the finite element method. The numerical scheme is based on the idea to represent the capillary bed as a network of one-dimensional channels that acts as a concentrated source of flow immersed into the interstitial volume, because of the natural leakage of capillaries. As a result, it can be classified as an embedded multiscale method. In the case of simple geometrical configurations of capillary vessels, such as an array of straight channels, semi-analytic solutions of the problem have been developed [13-15]. A more extensive application of numerical approximation methods has recently enabled the analysis of realistic microvascular geometries [2, 16-18]. Here, we extend this approximation strategy to problems involving blood flow and mass transport. The main advantage of the proposed scheme is that the computational grids required to approximate the equations on the capillary network and on the interstitial volume are completely independent. As a result, arbitrarily complex microvascular geometries may be potentially considered. From the standpoint of numerical approximation, the theoretical aspects of the method have been addressed in the works by D'Angelo [19-21].

Our results suggest that using nanoparticles as intermediate vectors for chemotherapy improves the treatment. For the same amount of injected dosage, drug charged nanoparticles provide higher concentration levels in the interstitial tissue of the tumor and more persistent delivery over time with respect to bolus injection. Thanks to the computational approach, these conclusions are based on the analysis of specific performance indicators, such as the interstitial drug concentration level, the drug metabolization rate, the cell surviving fraction and the corresponding timecourses.

\section{MATERIALS AND METHODS}

We start the derivation of the model by presenting the governing equations for microcirculation, tissue perfusion and mass transport. In a second phase, we will adapt these general equations to specific cases. The first case is the study of the coupled transport of oxygen and tirapazamine, a drug specifically designed to target hypoxic cells. In the second one, we apply the theory to analyze the delivery of drugs consequent to the injection of nanoparticles into the tumor region. 


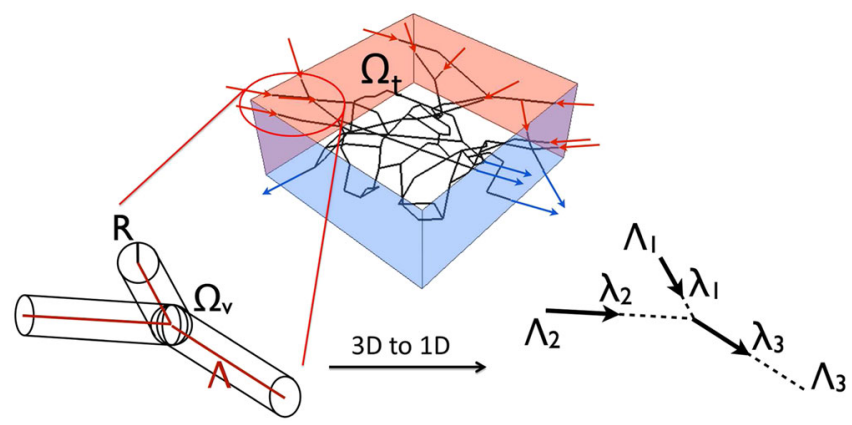

Figure 1. Visualization of a realistic vascular network that is used in the simulations, courtesy of Dr T. Secomb, available online at [22]. Below, we illustrate the transition from a three-dimensional representation of the vascular and interstitial domains, $\Omega_{v}, \Omega_{t}$ respectively, to the corresponding one-dimensional description of the vessels, where $\Omega$ denotes the interstitium and $\Lambda$ is the capillary network. The colors on the sides

of the tissue sample identify the inflow (red) and the outflow (blue) sections of the capillary network.

We aim to model fluid and mass transport in a permeable biological tissue perfused by a capillary network. We consider a domain $\Omega$ that is composed by two parts, $\Omega_{v}$ and $\Omega_{t}$, the capillary bed and the tumor interstitium, respectively. To account for the microvascular network, we model the capillaries as cylindrical vessels. We denote with $\Gamma$ the outer surface of $\Omega_{v}$, with $R$ its radius and with $\Lambda$ the centerline of the capillary network. A characteristic feature of the computational model is that the capillaries are actually represented as one-dimensional channels. As shown in [13-15, 17], this approximation significantly simplifies the problem at the computational level. This is performed by taking the limit $R \rightarrow 0$ and shrinking the capillary bed to its centerline $\Lambda$. We denote with $s$ the arc length coordinate along this line. A sketch of the domains before and after adopting the one-dimensional representation of the capillary network is visualized in Figure 1. After this step, we observe that the distinction between the subregion $\Omega_{t}$ and the entire domain $\Omega$ is no longer meaningful, because $\Lambda$ has null measure in $\mathbb{R}^{d}$. For notational convenience, in what follows, we will then identify $\Omega_{t}$ with $\Omega$ and $\Omega_{v}$ with $\Lambda$.

The physical quantities of interest are the flow pressure $p$, the velocity $\mathbf{u}$ and the concentration of transported solutes $c$. They are all defined as fields depending on time $t$ and space, being $\mathbf{x} \in \Omega$ the spatial coordinates. Furthermore, we denote with the subscript $v$ their restriction to the capillary bed (vessels), and with $t$ the restriction to the interstitial tissue. The derivation of our model stems from fundamental balance laws regulating the flow in the capillary bed, the extravasation of plasma and solutes and their transport in the interstitial tissue.

\subsection{Governing equations for flow and mass transport}

The flow model consists in two parts, the microcirculation and the flow in the interstitial volume, which interact through suitable interface conditions. We assume that the tumor interstitium behaves as an isotropic porous medium. The flow through the interstitium is modeled by the Darcy's law. A Newtonian model is applied for the blood flow in the capillaries. We want to take into account of the lymphatic drainage, which plays an important role in the phenomena we aim at studying [16, 23].

Microcirculation is an extreme case where the size of vessels is the smallest and the effect of blood pulsation is almost negligible. The Reynolds and the Womersley numbers characterizing the flow are very low if compared to other regions of the vascular network. As a result, Poiseuille's law for laminar stationary flow of incompressible viscous fluid appropriately describes this flow [4, 13]. Let us decompose the network $\Lambda$ into individual branches $\Lambda_{i}, i=1, \ldots, N$. We denote with $\lambda_{i}$ an arbitrary orientation of each branch that defines the increasing direction of the arc length $s_{i}$ (see also Figure 1). Let $\lambda, s$ be the same quantities referring to the entire network $\Lambda$.

One of the functions of the capillary network is to transport and distribute fluid and chemicals to the interstitial volume. This is achieved by means of the leakage of the capillary walls. We model this effect using the Kedem-Katchalsky equation, which expresses the volume flow $J_{b}$, 


$$
J_{b}:=L_{p}\left(\left(p_{v}-p_{t}\right)-\sum_{*} \sigma^{*}\left(\pi_{v}^{*}-\pi_{t}^{*}\right)\right)
$$

where $L_{p}$ is the hydraulic permeability of the vessel wall (see Table I for units and physiological values) and $p_{v}-p_{t}$ is the pressure difference between the vessels and the interstitial volume. Because of osmosis, the pressure drop across the capillary wall is affected by the difference in concentration of the chemicals dissolved in blood, [24, 25]. We denote quantities related to a generic solute carried by blood with the superscript $*$ and $\sum_{*}$ represents the summation over all the significant chemical species, as discussed below. Then, the oncotic pressure transmural gradient is $\pi_{v}^{*}-\pi_{t}^{*}$, where $\pi^{*}=R_{g} T c^{*}$ is the oncotic pressure given by a concentration $c^{*}$ of a given solute, being $R_{g}$ the universal gas constant and $T$ the absolute temperature. The oncotic pressure is modulated by the reflection coefficient $\sigma^{*}$ that quantifies the departure of a semipermeable membrane from the ideal permeability (where any molecule is able to travel across the membrane without resistance). Although the index $*$ spans over all solutes that are dissolved in blood, not all of them significantly affect the oncotic pressure. Only the large molecules, such as proteins, can induce a significant oncotic pressure gradient. Indeed, the oncotic pressure gradient is mainly due to the presence of albumin in blood, whose concentration can reasonably considered to be constant. According to data provided in [24, 25, 27], the oncotic pressure gradient due primarily to albumin in arterioles and capillaries is about $25 \mathrm{mmHg}$, which is comparable to the hydrostatic pressure in the vessel. In contrast, we assume that solutes such as oxygen or low concentrated drugs cannot significantly contribute. This assumption will be further discussed in what follows, on the basis of the physical parameters characterizing the transport of the considered solutes (Tables I and II). As a result, for our purposes, the capillary leakage only depends on the hydrostatic pressure according to the following expression,

$$
J_{b}:=L_{p}\left(\left(p_{v}-p_{t}\right)-\sigma^{p}\left(\pi_{v}^{p}-\pi_{t}^{p}\right)\right)=L_{p}\left(\left(p_{v}-p_{t}\right)-\sigma^{p} R_{g} T\left(c_{v}^{p}-c_{t}^{p}\right)\right),
$$

Table I. Physical parameters characterizing the perfusion problem.

\begin{tabular}{llcccc}
\hline Symbol & \multicolumn{1}{c}{ Parameter } & Units & Value & Source & Equation \\
\hline$L_{p}$ & Hydraulic permeability, capillary wall & $\mathrm{m}^{2} \mathrm{~s} / \mathrm{kg}$ & $10^{-10}$ & {$[26]$} & $(1)$ \\
$\sigma^{p} \pi_{v}^{p}$ & Vessel oncotic pressure & $\mathrm{mmHg}$ & 28 & {$[25]$} & $(1)$ \\
$\sigma^{p} \pi_{t}^{p}$ & Interstitial oncotic pressure & $\mathrm{mmHg}$ & 0.1 & {$[25]$} & $(1)$ \\
$L_{p}^{L F} S$ & Effective permeability, lymphatic vessels & $(\mathrm{mmHg} \mathrm{hour})^{-1}$ & 0.5 & {$[5]$} & $(2 \mathrm{a})$ \\
$\kappa$ & Hydraulic permeability, interstitial volume & $\mathrm{m}^{2}$ & $10^{-18}$ & {$[16]$} & $(2 \mathrm{~b})$ \\
$\mu$ & Blood viscosity & $\mathrm{kg} /(\mathrm{ms})$ & $4 \times 10^{-3}$ & {$[16]$} & $(2 \mathrm{~b})$ \\
$\mathrm{R}$ & Capillary radius & $\mu \mathrm{m}$ & 7.64 & {$[22]$} & $(2 \mathrm{c})$ \\
$p_{0}+\Delta p$ & Inflow pressure & $\mathrm{mmHg}$ & 35 & {$[25]$} & $(5)$ \\
$\Delta p$ & Axial pressure drop & $\mathrm{mmHg}$ & 1.25 & {$[16]$} & $(5)$ \\
$\beta_{b}$ & Boundary conductivity & $\mathrm{m}{ }^{2} \mathrm{~s} / \mathrm{kg}$ & $5 \times 10^{-11}$ & {$[16]$} & $(7)$ \\
\hline
\end{tabular}

\begin{tabular}{|c|c|c|c|c|c|c|c|}
\hline Symbol & Parameter & Units & \multicolumn{2}{|c|}{ Oxygen } & \multicolumn{2}{|l|}{ TPZ } & Equation \\
\hline$P$ & Vascular permeability & $\mathrm{cm} / \mathrm{s}$ & $3.5 \times 10^{-3}$ & {$[17]$} & $5 \times 10^{-3}$ & 2.2 .1 & (3) \\
\hline$D_{t}$ & Interstitial diffusivity & $\mathrm{cm}^{2} / \mathrm{s}$ & $1.35 \times 10^{-5}$ & {$[2]$} & $1.87 \times 10^{-6}$ & {$[2]$} & (4) \\
\hline$D_{v}$ & Vascular diffusivity & $\mathrm{cm}^{2} / \mathrm{s}$ & $5 \times 10^{-3}$ & 2.2 .1 & $6.926 \times 10^{-4}$ & 2.2 .1 & (4) \\
\hline$C_{\max }^{t p z}$ & Inflow concentration & $\mathrm{g} / \mathrm{m}^{3}$ & - & - & 48 & {$[2]$} & (6) \\
\hline$C_{\max }^{\text {max }}$ & Inflow concentration & $\mathrm{mmHg}$ & 100 & {$[28]$} & - & - & (6) \\
\hline$\beta_{c}$ & Boundary boundary & $\mathrm{m} / \mathrm{s}$ & $10^{-5}$ & {$[16]$} & $10^{-5}$ & [16] & (8) \\
\hline$m_{0}^{t p z}$ & Metabolization rate & $\mathrm{s}^{-1}$ & - & - & 0.0317 & {$[2]$} & (9) \\
\hline$m_{0}^{o x}$ & Metabolization rate & $\mathrm{mmHg} / \mathrm{s}$ & 8.0645 & [2] & - & - & (10) \\
\hline
\end{tabular}

Table II. Physical parameters for oxygen and TPZ delivery, transport and metabolization. 
where $*=p$ denotes the contribution of proteins to the oncotic pressure and, in agreement with the definition of $\pi^{p}, c_{v}^{p}$ and $c_{t}^{p}$ denote the constant protein concentration in the capillaries and the interstitial tissue respectively. As a consequence, the flow equations do not depend on the ma transport model that will be developed in the next section.

To balance capillary leakage, the venous and the lymphatic systems absorb the fluid in excess. Following [5] and [23], we model them as a distributed sink term in the equation for the tissue perfusion. More precisely, we assume that the volumetric flow rate due to lymphatic vessels, $\Phi^{L F}$, is proportional to the pressure difference between the interstitium and the lymphatics, namely $\Phi^{L F}\left(p_{t}\right)=L_{p}^{L F} \frac{S}{V}\left(p_{t}-p_{L}\right)$, where $L_{p}^{L F}$ is the hydraulic permeability of the lymphatic wall, $S / V$ is the surface area of lymphatic vessels per unit volume of tissue and $p_{L}$ is the hydrostatic pressure within the lymphatic channels. The values of these parameters with the corresponding units are listed in Table I. The coupled problem for microcirculation and perfusion consists to find the pressure fields $p_{t}, p_{v}$ and the velocity fields $\mathbf{u}_{t}, \mathbf{u}_{v}$ such that the flow problem is

$$
\begin{cases}-\nabla \cdot\left(\frac{\kappa}{\mu} \nabla p_{t}\right)+L_{p}^{L F} \frac{S}{V}\left(p_{t}-p_{L}\right)-f_{b}\left(\bar{p}_{t}, p_{v}\right) \delta_{\Lambda}=0 & \text { in } \Omega, \\ \mathbf{u}_{t}=-\frac{\kappa}{\mu} \nabla p_{t} & \text { in } \Omega, \\ -\frac{\pi R^{4}}{8 \mu} \frac{\partial^{2} p_{v}}{\partial s^{2}}+f_{b}\left(\bar{p}_{t}, p_{v}\right)=0 & s \in \Lambda, \\ \mathbf{u}_{v}=-\frac{R^{2}}{8 \mu} \frac{\partial p_{v}}{\partial s} \lambda & s \in \Lambda,\end{cases}
$$

where $\kappa$ is the hydraulic permeability of the interstitial volume, $\mu$ is the blood viscosity and the function $f_{b}\left(\bar{p}_{t}, p_{v}\right) \delta_{\Lambda}$ denotes the fluid flux leaking from the capillaries to the interstitial volume and it is defined by,

$$
f_{b}\left(\bar{p}_{t}, p_{v}\right):=2 \pi R L_{p}\left(\left(p_{v}-\bar{p}_{t}\right)-\sigma^{p}\left(\pi_{v}^{p}-\bar{\pi}_{t}^{p}\right)\right),
$$

while the symbol $\delta_{\Lambda}$ in (2a) is the Dirac measure located on $\Lambda$. For an appropriate dimensional interpretation of (2a), we remind that $\delta_{\Lambda}$ is not a dimensionless function. According to its definition $\int_{\Omega} f \delta_{\Lambda} d \mathbf{x}=\int_{\Lambda} f d s$, the dimension of $\delta_{\Lambda}$ is [length $]^{-2}$, where $d \mathbf{x}$ is a volume element in the interstitial space with coordinate vector $\mathbf{x}$. Equation (2c) represents Poiseuille flow on the capillary network. Because the capillary bed is modeled as a one-dimensional network embedded into the interstitial volume, the equations would be ill posed if the coupling between the two subregions was considered pointwise $[20,21]$. For this reason, the function $f_{b}\left(\bar{p}_{t}, p_{v}\right)$ is such that the capillary bed is affected by the average of quantities in the interstitial tissue, calculated on a cylindrical surface that represents the actual size of capillaries (see Figure 1 for a sketch). The average value of pressure, velocity or concentration fields, denoted by general function $g$, over the real surface of the capillary bed is denoted by

$$
\bar{g}(s):=\frac{1}{2 \pi R} \int_{0}^{2 \pi} g(s, \theta) R d \theta .
$$

For a more detailed derivation of this model from the problem formulation where also the capillaries are modeled as three-dimensional channels, we refer the interested reader to [16].

To model drug transport in the interstitial tissue, we assume that molecules are advected by the fluid and diffuse in all $\Omega$. In addition, chemical species may be metabolized by the cells in the interstitial tissue. The distribution of solutes in the interstitial tissue is also affected by the lymphatic drainage. According to the assumptions at the basis of the flow model, the effect of lymphatic drainage on mass transport is described as a distributed sink proportional to $L_{p}^{L F} \frac{S}{V}\left(p_{t}-p_{L}\right) c_{t}$. 
Mass transport in the capillary bed is modeled by means of advection-diffusion equations. As shown in [19], the one-dimensional model for mass transport in the capillaries network can be derived starting from the actual 3D advection-diffusion problem. The coupled problem, accounting for transport of chemicals from the microvasculature to the interstitium, consists to find the concentrations $c_{v}$ and $c_{t}$ respectively, such that the general mass transport problem is

$$
\begin{cases}\frac{\partial c_{v}}{\partial t}+\frac{\partial}{\partial s}\left(\left(\mathbf{u}_{v} \cdot \lambda\right) c_{v}-D_{v} \frac{\partial c_{v}}{\partial s}\right)=-\frac{1}{\pi R^{2}} f_{c}\left(\bar{p}_{t}, p_{v}, \bar{c}_{t}, c_{v}\right) & \text { on } \Lambda,(4 \mathrm{a}) \\ \frac{\partial c_{t}}{\partial t}+\nabla \cdot\left(c_{t} \mathbf{u}_{t}-D_{t} \nabla c_{t}\right)+m c_{t}+L_{p}^{L F} \frac{S}{V}\left(p_{t}-p_{L}\right) c_{t}=f_{c}\left(\bar{p}_{t}, p_{v}, \bar{c}_{t}, c_{v}\right) \delta_{\Lambda} & \text { in } \Omega,(4 \mathrm{~b})\end{cases}
$$

where $D_{v}$ and $D_{t}$ are the molecular diffusivities, in the capillaries and the interstitium, respectively, assumed to be constant in each region. The rate of metabolization in the interstitium is denoted by $m$. This parameter may in turn be a function of the concentrations, as will be pointed out later on (see Table II for values and units). The function $f_{c}\left(\bar{p}_{t}, p_{v}, \bar{c}_{t}, c_{v}\right)$ is a mass flux per unit length of the capillary vessels, and it accounts for the mass transfer from the capillary bed to the interstitial tissue. The concentration in the vascular network, $c_{v}$, is defined as mass per unit volume; therefore, the linear concentration is given by $A c_{v}$, being $A=\pi R^{2}$ the cross sectional area of a blood vessel. In order to restore the dimensional homogeneity of (4a) and (4b), we divide all the terms of (4a) by $\pi R^{2}$. As a result, the factor $\left(\pi R^{2}\right)^{-1}$ multiplies the last term of (4a). We describe the capillary walls as semipermeable membranes allowing not only for the leakage of fluid but also for the selective filtration of molecules. Again, the Kedem-Katchalsky equations represent a good model for these phenomena [25]. According to these equations, the flux of chemicals per unit surface across the capillary walls is

$$
J_{c}:=(1-\sigma) J_{b}\left(p_{t}, p_{v}\right) c_{t / v}+P\left(c_{v}-c_{t}\right) \text { on } \Gamma,
$$

where $P$ is the permeability of the vessel wall with respect to solutes, $J_{b}$ is defined in (1) and $\sigma$ is the osmotic reflection coefficient. It quantifies the departure of the membrane behavior from the case of ideal permeability. The symbol $c_{t / v}$ denotes the average concentration within the capillary walls. It is defined as a suitable combination of the concentrations on the two sides of the walls [27]. In particular, we set $c_{t / v}:=w \bar{c}_{t}+(1-w) c_{v}$ where $0<w<1$ is a weight that depends on the Péclét number of the solute transport through the wall. Then, under the assumption that capillaries can be modeled as cylindrical channels, the magnitude of the mass flux exchanged per unit length between the network of capillaries and the interstitial volume at each point of the capillary vessels is the following:

$$
f_{c}\left(\bar{p}_{t}, p_{v}, \bar{c}_{t}, c_{v}\right)=2 \pi R\left[(1-\sigma) J_{b}\left(\bar{p}_{t}, p_{v}\right) c_{t / v}+P\left(c_{v}-\bar{c}_{t}\right)\right] .
$$

2.1.1. Boundary and initial conditions. The fluid dynamics and mass transport equations are not complete yet. Before being solved, they must be complemented with boundary conditions on the artificial sections that separate the domains $\Omega$ and $\Lambda$ from the surrounding tissue. We model a sample of tissue that is able to exchange fluid and mass with the exterior. In addition, for the governing equations that depend on time, we need to prescribe the initial conditions of the system. Only the drug transport equations depend on time. The initial drug concentrations will be set to the basal values, equal to zero.

For the prescription of boundary conditions on the capillary network, we have to define what are the inflow and outflow boundaries. As shown in Figure 1 using different colors and arrows, we denote by $\partial \Lambda_{\text {in }}$ and $\partial \Lambda_{\text {out }}$, the inflow and outflow sections of $\partial \Lambda$, respectively. We regulate the flow by enforcing the values of the blood pressure at the extrema of the capillaries. As a result, we prescribe the following conditions:

$$
p_{v}=p_{0}+\Delta p \text { on } \partial \Lambda_{\text {in }} \text { and } p_{v}=p_{0} \text { on } \partial \Lambda_{\text {out }},
$$

where the total pressure drop $\Delta p$ is computed to ensure that the average blood velocity in the network fits with the measured values in healthy human microvasculature [16]. In order to model 
the administration of the drug through the vascular system, we assume that a fixed drug concentration, denoted with $c_{v, \max }$, is injected in the blood stream for a period of time $t \in(0, T)$. We then enforce

$$
c_{v}=c_{v, \text { max }} \text { if } t \in(0, T), c_{v}=0 \text { otherwise, on } \partial \Lambda_{\text {in }} \text { and } \frac{\partial c_{v}}{\partial s}=0 \text { on } \partial \Lambda_{\text {out }} .
$$

On the outflow boundary of the network, we constrain the derivatives of the drug concentration, rather than the value itself. As a consequence, the concentration value is determined by the model, on the basis of the convection and reaction mechanisms.

The interstitial tissue, $\Omega$, is assumed to be an isotropic material. To comply with this property, we enforce on all the artificial interfaces of the tissue, $\partial \Omega$, boundary conditions that mimic the resistance of the surrounding material. For the fluid dynamics equations, these conditions are discussed in detail in [16] and read as follows:

$$
-\frac{\kappa}{\mu} \nabla p_{t} \cdot \mathbf{n}=\beta_{b}\left(p_{t}-p_{0}\right)
$$

where $\mathbf{n}$ is the outer unit normal vector of the interstitial boundary, $p_{0}$ is the basal (atmospheric) pressure and $\beta_{b}$ is a parameter inversely proportional to the resistance of the surrounding tissue. Equation (7) is very general. When $\beta_{b} \rightarrow 0$ it corresponds to infinite resistance. In this case, the fluid flow cannot cross the boundaries of $\Omega$. Conversely, large values of $\beta_{b}$ (asymptotically $\beta_{b} \rightarrow \infty$ ) correspond to enforcing $p_{t}=p_{0}$ on the boundaries. The sensitivity of the solution with respect to $\beta_{b}$ and suitable expressions to calculate it on the basis of the other model parameters are provided in [16]. We proceed analogously for the transport of solutes by setting,

$$
-D_{t} \nabla c_{t} \cdot \mathbf{n}=\beta_{c} c_{t},
$$

where $\beta_{c}$ quantifies the permeability of the outer tissue with respect to solute transport. Here, we have implicitly assumed that the basal solute concentration is equal to zero. The values of $\beta_{b}, \beta_{c}$ used in the simulations are reported in Tables I and II, respectively.

\subsection{Coupled system of $\mathrm{O}_{2}$ and tirapazamine}

Hypoxia targeted drugs, such as tirapazamine (TPZ), are designed to be metabolized more quickly by hypoxic cells. The distribution of such drugs in the interstitial tissue then depends on the local availability of oxygen. Mathematical models and simulations have already been applied to study these effects [2]. The objective of this section is to adapt the single-specie mass transport model developed above to the case of multiple solutes, in order to reproduce the study presented in [2], with a more detailed mathematical description.

We study the distribution of the oxygen concentration, denoted by $c^{o x}$. Because oxygen is persistently supplied by the capillary bed, we rely on the steady problem formulation. Oxygen distributes into the interstitial volume thanks to diffusion and transport. The rate of oxygen absorption depends in turn on the oxygen concentration profile itself. This dependence is represented by a Michaelis-Menten formula [25],

$$
m^{o x}\left(c_{t}^{o x}\right)=\frac{m_{0}^{o x}}{c_{t}^{o x}+c_{0}^{o x}},
$$

where $m_{0}^{o x}$ represents the maximal oxygen demand, that is, the rate of oxygen consumption when oxygen is not limited, and $c_{0}^{o x}$ is the oxygen concentration at which the reaction rate is half of $m_{0}^{o x}$. Let us now denote by $c^{t p z}$ the concentration of TPZ. This is a relatively small molecule that obeys to the governing equations of mass transport described in (4a). Following [2], the consumption rate of TPZ depends on the oxygen concentration through the following expression

$$
m^{t p z}\left(c_{t}^{o x}\right)=m_{0}^{t p z} \frac{c_{0}^{t p z}}{c_{0}^{t p z}+c_{t}^{o x}},
$$


where $m_{0}^{t p z}$ is the metabolization rate when TPZ metabolism is not limited by the oxygen concentration, and $c_{0}^{t p z}$ is the oxygen concentration at which the consumption rate for TPZ is halved compared to that under anoxia. According to Table II, the dimensions of the metabolization kinetic terms $m^{o x}\left(c_{t}^{o x}\right)$ and $m^{t p z}\left(c_{t}^{o x}\right)$ are $[t i m e]^{-1}$, which make them match with the other terms of (11a) and (11b), respectively.

The general mass transport model (4), adapted to the previous assumptions, ends up with the following equations,

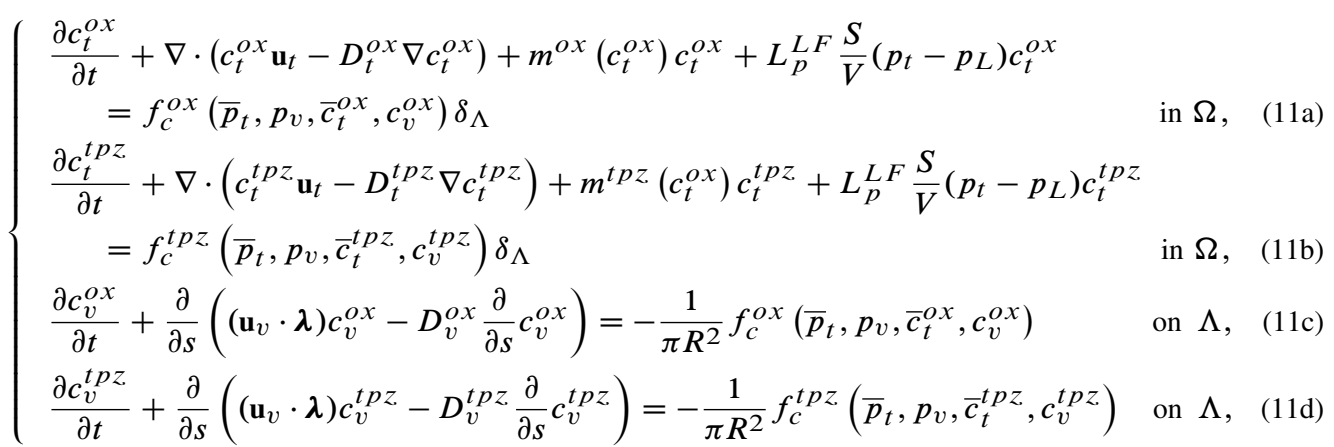

where the flux of the specie $*=o x, t p z$ across the capillary wall is defined by the function,

$$
f_{c}^{*}\left(\bar{p}_{t}, p_{v}, \bar{c}_{t}^{*}, c_{v}^{*}\right)=2 \pi R\left[\left(1-\sigma^{*}\right) L_{p}\left(\left(p_{v}-\bar{p}_{t}\right)-\sigma^{p}\left(\pi_{v}^{p}-\bar{\pi}_{t}^{p}\right)\right) c_{t / v}^{*}+P^{*}\left(c_{v}^{*}-\bar{c}_{t}^{*}\right)\right] .
$$

2.2.1. Parameters of the model. We apply the coupled oxygen-TPZ model, namely (11a)-(11d), to calculate the time and space dependent concentration profiles of TPZ in the interstitial volume, after a bolus injection of TPZ equal to $C_{\max }^{t p z}$ for a duration of $T_{\max }=20 \mathrm{~min}$. The numerical simulation is however extended for a longer time interval. As a result, we enforce the following time dependent boundary condition for TPZ, $c_{v}^{t p z}=C_{\text {max }}^{t p z}$ for $t \in\left(0, T_{\text {max }}\right)$ and $c_{v}^{t p z}=0$ for $t>T_{\text {max }}$ on $\partial \Lambda_{i n}$, while we assume that oxygen is flowing steadily into the tissue sample, according to the boundary condition $c_{v}^{o x}=C_{\max }^{o x}$ on $\partial \Lambda_{i n}$. The values of $C_{\text {max }}^{t p z}$ and $C_{\text {max }}^{o x}$ are shown in Table II. In particular, $C_{\max }^{o x}$ is set to the value of saturated blood, which corresponds to an oxygen partial pressure of $100 \mathrm{mmHg}$. The parameters needed to feed the fluid dynamics, and the mass transport equations are taken from different sources, reported in Tables I and II. In particular, we observe that for $D_{v}^{o x}$ and $D_{v}^{t p z}$, we do not refer the reader to previously publishes studies, because the estimate of these parameters needs a special discussion. Concerning the diffusivity of oxygen in the blood stream, $D_{v}^{o x}$, the value of Table II is significantly larger than the usual diffusivity for oxygen transport in arteries. Indeed, in [29], the authors and coworkers have used $D_{v}^{o x} \simeq 10^{-5} \mathrm{~cm}^{2} / \mathrm{s}$. Diffusion of oxygen was on purpose augmented in the present work in order to compensate for the role of red blood cells and in particular for the additional oxygen available because of the exchange of dissolved gas and hemoglobin-bound oxygen. Because we cannot model this interaction using the mass transport (4), we take it into account approximately by augmenting the oxygen diffusivity. This is an alternative way to increase the availability of oxygen in the capillary network. The magnitude of the augmented oxygen diffusion coefficient was determined by an heuristic approach consisting in the comparison of our numerical simulations with the ones reported in [17] (see in particular Figure 3), where the oxygen concentration maps in the interstitial tissue are calculated for the same vascular geometry of this work, but using a different oxygen transport model accounting for hematocrit discharge and hemoglobin saturation.

Concerning TPZ, the value of $D_{v}^{t p z}$ shown in Table II is based on the combination of StokesEinstein equation with the available diffusivity of TPZ in the interstitial volume and the diffusivities of oxygen. More precisely, given the Stokes-Einstein model,

$$
D=\frac{K_{B} T}{6 \pi \mu r}
$$


where $r$ is an estimate of the molecular radius, $k_{B} T$ is the Boltzmann thermal energy and $\mu$ is the viscosity of the solvent, we assume that it is adequate to estimate oxygen and TPZ diffusion in the blood stream and the interstitial volume. As a result, the following proportion holds true

$$
D_{v}^{t p z}=D_{t}^{t p z} \frac{D_{v}^{o x}}{D_{t}^{o x}}
$$

We observe that the augmentation factor discussed for oxygen applies to the diffusivity of TPZ in blood as well. To assess the sensitivity of the computational results with respect to the magnitude of $D_{v}^{t p z}$, we have performed an additional simulation of the TPZ concentration maps in the capillary network and in the interstitial volume using $D_{v}^{t p z}=6.926 \times 10^{-6} \mathrm{~cm}^{2} / \mathrm{s}$, which is decreased of two orders of magnitude with respect to the value of Table II. The corresponding results (not shown) were compared with the ones obtained with $D_{v}^{t p z}=6.926 \times 10^{-4} \mathrm{~cm}^{2} / \mathrm{s}$. This analysis confirms that the magnitude of $D_{v}^{t p z}$ only mildly affects the concentration maps in the interstitial tissue, essentially because the distribution of TPZ in the capillary network is transport dominated.

Before proceeding, we aim to use the available data to verify the assumption that the contribution of oxygen and TPZ to the oncotic pressure is negligible. This hypothesis has already been widely investigated for oxygen, [27,30], and it results to be accurately satisfied, because oxygen is a very small molecule. For TPZ, the question remains open. An upper bound for the oncotic pressure generated by TPZ dissolved in blood is $\pi_{\text {max }}^{t p z}=\sigma^{t p z} R_{g} T C_{\text {max }}^{t p z}$. The main issue is the quantification of the reflection coefficient $\sigma^{t p z}$. According to [24, 25, 27] and using (12), this parameter can be estimated as

$$
\left.\sigma^{t p z}=1-\left(1-\frac{r^{t p z}}{r^{\text {pores }}}\right)^{2}\right)^{2} \quad \text { and } \quad r^{t p z}=\frac{k_{B} T}{6 \pi \mu D_{t}^{t p z}},
$$

where $r^{t p z}$ is an estimate of the $T P Z$ molecular radius and $r^{\text {pores }}$ quantifies the average dimension of the endothelial fenestrations in the capillary walls. For the latter, following [27] and references therein, we take $r^{\text {pores }}=5 \times 10^{-9} \mathrm{~m}$. For the former, we use the Stokes-Einstein equation to approximate the TPZ radius using the molecule diffusivities, provided in Table II. This results in the following upper bound for the TPZ radius $r^{t p z}<3 \times 10^{-10} \mathrm{~m}$. When we compare this estimate with the Bohr radius (the most probable distance between the proton and electron in a hydrogen atom), it turns out that one TPZ molecule should span approximately over 5 radii, which seems to be appropriate for the molecule, whose chemical formula is $\mathrm{C}_{7} \mathrm{H}_{6} \mathrm{~N}_{4} \mathrm{O}_{2}$. Using the available estimate for $r^{t p z}$, we obtain $\sigma^{t p z}<0.013$, which completely justifies our assumption. Indeed, the corresponding oncotic pressure is $\pi_{\text {max }}^{t p z}=\sigma^{t p z} R_{g} T C_{\text {max }}^{t p z}<0.08 \mathrm{mmHg}$. This value is almost negligible with respect to the oncotic pressure gradient induced by the blood proteins that amounts to $25 \mathrm{mmHg}$.

\subsection{Transport of nanoparticles and drug delivery}

Nanoparticles are used as vectors for the delivery of drugs to the tumor tissue. The advantage of this technology with respect to systemic delivery is that chemotherapy agents are released selectively to the tumor mass [31]. The side effects of drugs on patients health are thus reduced. We aim at modeling the transport of nanoparticles in the capillary network and the consequent delivery of a drug, which in our case is again TPZ, to enable comparisons with the bolus injection delivery method. A large variety of cancer treatment methods based on nanoparticle delivery is available or under development [32]. Thanks to its generality, the computational model could be adapted to describe many of them. Here, we focus on modeling drug delivery from particles designed to travel the vascular network and selectively interact with particular receptors on the capillary walls. Mathematical models describing these phenomena have been recently proposed, we refer to [33, 34]. They are based on similar concepts. In the former study, nanoparticle adhesion to tumor vasculature is addressed. The latter one is devoted to study the interaction of nanoparticles with the coronary arteries, in presence of regions affected by inflammation. Our model arises from the general equations of blood flow and mass transport, with some modifications. In particular, it has to be adapted to account 
for three different stages of the delivery process: (i) the transport of nanoparticles in the capillary network; (ii) the adhesion of the particles to the capillary wall, adopting the framework proposed in $[33,34]$; and (iii) the delivery of the encapsulated drug to the surrounding tissue. Given the moderately short time scales addressed in the forthcoming numerical examples, as well as the complexity of the biological phenomena involved, many of which are not completely understood yet, we do not consider modeling of nanoparticles extravasation and migration into the interstitial volume.

Steps (i) and (ii): nanoparticle transport and adhesion. The model accounting for nanoparticle transport in the blood stream and their adhesion to the wall results in the following equations:

$$
\frac{\partial c_{v}}{\partial t}+\frac{\partial}{\partial s}\left(\left(\mathbf{u}_{v} \cdot \lambda\right) c_{v}-D_{v} \frac{\partial c_{v}}{\partial s}\right)+\frac{2 \pi R}{\pi R^{2}} \Pi c_{v}=0 \quad \text { in } \Lambda \times(0, T),
$$

where $c_{v}(\mathbf{x}, t)$ is the nanoparticle concentration inside the vessels and it is measured as number of particles per unit volume $\left[\sharp / \mathrm{m}^{3}\right]$. The adhesion of particles to the wall, which was not accounted in the general model, is described as a sink term distributed along the length of the capillary network, namely $\Pi c_{v}$ on $\Lambda$. The new term $\Pi c_{v}$ is a flux of particles sequestrated to the flow per unit surface of capillary wall. Because we consider a one-dimensional model along the capillary axis, we use the corresponding flux per unit length $2 \pi R \Pi c_{v}$. The sink term, per unit volume, equivalent to this flux is then obtained by scaling the flux per unit length with the vessel cross section, that is $\pi R^{2}$. The vascular deposition parameter, $\Pi$, is estimated using a ligand-receptor model for the interaction of particles with the endothelial layer. In particular, we use the model setting of [34]. For the sake of completeness, we report here the main components of the model. The vascular deposition parameter is defined as

$$
\Pi(s)=\mathcal{P}_{a}|S(s)| \frac{d_{p}}{2},
$$

where $\mathcal{P}_{a}$ is the probability of particle adhesion, $S(s)$ is the wall shear rate and $d_{p}$ is the diameter of the considered nanoparticles. Given the plasma viscosity $\mu$, the wall shear stress at the axial coordinate $s$ along the capillary network is $\mu S(s)$. As a result, we compute the wall shear rate using the Poiseuille's flow equation. To this aim, we remind that the network $\Lambda$ can be decomposed into individual branches $\Lambda_{i}, i=1, \ldots, N$. Then, the shear rate assumes a constant value on each branch given by

$$
\left|S_{i}\right|=\frac{R}{2 \mu} \frac{\left|\Delta_{i} p_{v}\right|}{L_{i}},
$$

where $\left|\Delta_{i} p_{v}\right|$ is the absolute value of the pressure drop along each branch of the network and $L_{i}$ is the branch length. The probability of adhesion, $\mathcal{P}_{a}$, is in turn defined as a function of particle size, shape and surface properties,

$$
\mathcal{P}_{a}(s)=m_{l} K_{a}^{0} \alpha_{2} \pi r_{0}^{2} \exp \left(-\beta \frac{\mu|S(s)|}{\alpha_{2}}\right) .
$$

In the above expression, $m_{l}$ is the surface density of the ligand molecules that decorate the nanoparticle surface and $K_{a}^{0}$ is the affinity constant of the interaction between ligands and receptors. The parameter $\alpha_{2}$, defined as

$$
\alpha_{2}=m_{r}\left[1-\left(1-\frac{\Delta}{d_{p} / 2}\right)^{2}\right]
$$

is a function of the density of receptors on the endothelial surface, $m_{r}$, and of the separation distance between the particle and the substrate at the equilibrium, $\Delta$. The parameter $r_{0}$ represents the radius of the adhesion point and $\beta=\frac{6 F \lambda}{k_{B} T}$ is a constant, where $\lambda$ is a characteristic length of the ligandreceptor bond, $F$ is the coefficient of hydrodynamic drug force on the spherical particle and $k_{B} T$ is the Boltzmann thermal energy. 
The model must be complemented by suitable initial and boundary conditions. At the inlet $\partial \Lambda_{\text {in }}$ we prescribe a Dirichlet boundary condition $c_{v}^{0}$, which represents the amount of injected particles. At the outflow $\partial \Lambda_{\text {out }}$ we specify a homogeneous Neumann boundary condition. We assume that the blood stream does not contain any particle at the initial time.

Once the problem for particle transport and adhesion is solved, we compute the density of nanoparticles adhering per unit surface to the wall as

$$
\Psi(s, t):=\int_{0}^{t} \Pi(s) c_{v}(s, \tau) d \tau .
$$

Step (iii): drug release from nanoparticles. The particles decorating the capillary wall are loaded with drug, and they are able to release it to the surrounding tissue. Observing that the particles are in direct contact with the capillary wall, we assume that the drug release rate to the interstitial volume per unit capillary surface is determined by the combination of the flux delivered by a single particle with the density of particles adhering to the wall. Determining the release profile of a single (spherical) loaded particle is a well studied problem in pharmacology [35]. Here, following [35-37], we define it using a power law model with saturation,

$$
\frac{q(t)}{q_{\infty}}=\frac{t^{b}}{t^{b}+\Upsilon}, \quad q_{\infty}=c_{n p}^{*} V_{n p}, \quad \text { then } \quad q(t)=\frac{t^{b}}{t^{b}+\Upsilon} c_{n p}^{*} V_{n p},
$$

where $q(t)$ is the amount of drug released and $q_{\infty}$ is the total drug load of a nanoparticle, given by the total drug concentration inside the nanoparticle, $c_{n p}^{*}$ (where $*$ denotes an unspecified drug loaded on the particles), multiplied by the nanoparticle volume $V_{n p}$. The saturation parameter $\Upsilon$ is expressed in dimensions of $[\text { time }]^{b}$. The two parameters $\Upsilon$ and $b$ reflect the structural and geometric properties of the delivery system. The drug release rate from a single nanoparticle is therefore obtained as

$$
J_{n p}(t)=\frac{d q(t)}{d t}=\frac{\Upsilon \cdot b \cdot t^{b-1}}{\left(t^{b}+\Upsilon\right)^{2}} c_{n p}^{*} V_{n p},
$$

and the total drug release rate per unit surface is computed as,

$$
J(s, t)=J_{n p}(t) \Psi(s, t),
$$

where $\Psi(s, t)$ is the density of nanoparticles adhering per unit surface to the wall.

To conclude, we apply the immersed boundary method to describe the capillary bed as a source term concentrated on the centerline $\Lambda$. More precisely, the action of drug loaded nanoparticles on the interstitial tissue is described by the source term $2 \pi R J(s, t)$, assuming that the capillaries are cylindrical channels of radius $R$. To compare the nanoparticle delivery approach with the bolus delivery strategy previously considered, we load the particles with TPZ. The drug concentration into the tissue is modeled by the following equations and Robin (Neumann for $\beta_{c}=0$ ) boundary condition:

$$
\left\{\begin{array}{rr}
\frac{\partial c_{t}^{t p z}}{\partial t}+\nabla \cdot\left(c_{t}^{t p z} \mathbf{u}_{t}-D_{t}^{t p z} \nabla c_{t}^{t p z}\right)+m^{t p z}\left(c_{t}^{o x}\right) c_{t}^{t p z} & \\
+L_{p}^{L F} \frac{S}{V}\left(p_{t}-p_{L}\right) c_{t}^{t p z}=2 \pi R J(t) \delta_{\Lambda} & \text { in } \Omega \times(0, T], \\
D_{t}^{t p z} \nabla c_{t}^{t p z} \cdot \mathbf{n}=\beta_{c}\left(c_{t}^{t p z}-c_{0}^{t p z}\right) & \text { on } \partial \Omega \times(0, T] .
\end{array}\right.
$$

2.3.1. Parameters of the model. The nanoparticle transport and adhesion model requires to characterize several parameters, for which we refer to Table 1 in [34]. Furthermore, it is possible to calibrate the power law model in order to describe different scenarios, for example a fast release mechanism or a slow release rate. We fix the parameters of the model, $\Upsilon$ and $b$, such that $90 \%$ of the total drug is released within one day. The parameter values characterizing particle adhesion and drug release are reported in Table III. 
Table III. Physical parameters used to model nanoparticle injection and adhesion [34].

\begin{tabular}{llccc}
\hline Symbol & \multicolumn{1}{c}{ Parameter name } & Units & Value & Equation \\
\hline$D_{v}$ & Nanoparticle vascular diffusivity & $\mathrm{cm}^{2} / \mathrm{s}$ & $6.98 \times 10^{-9}$ & $(13)$ \\
$d_{p}$ & Nanoparticle diameter & $\mathrm{m}$ & $2 \times 10^{-6}$ & $(14)$ \\
$\beta$ & - & $\mathrm{N}^{-1}$ & $2.39 \times 10^{11}$ & $(15)$ \\
$\alpha_{2}$ & - & $\sharp / \mathrm{m}^{2}$ & $3.4 \times 10^{9}$ & $(15)$ \\
$m_{l} K_{a}^{0} r_{0}^{2}$ & - & $\mathrm{m}^{2}$ & $1.2585 \times 10^{-9}$ & $(15)$ \\
$\mathrm{b}$ & Power law exponent & - & 0.8 & $(17)$ \\
$\Upsilon$ & Power law saturation parameter & $\mathrm{hours}^{b}$ & 1 & $(17)$ \\
$C_{\text {max }}^{n p}$ & Inflow nanoparticle concentration & $\sharp / \mathrm{m}^{3}$ & $1.4354 \times 10^{12}$ & $(19)$ \\
\hline
\end{tabular}

Another important quantity is the concentration of nanoparticles injected at the inflow of capillary network. Because we are interested in comparing the amount of TPZ delivered from bolus and nanoparticle injection, we aim at determining the concentration of injected nanoparticles that match the TPZ bolus concentration, previously defined as $C_{\max }^{t p z}$. Similarly, the concentration of injected nanoparticles will be denoted by $C_{\text {max }}^{n p}$, and its value is determined according to the following balance equation:

$$
C_{\text {max }}^{n p} c_{n p}^{t p z} V_{n p}=C_{\text {max }}^{t p z}
$$

To determine the value of $C_{\max }^{n p}$, we need an estimate of the amount of drug cast in each particle, namely $c_{n p}^{t p z}$. To determine this value, we rely on two assumptions: (i) the drug mass fraction in each particle, denoted as $f^{t p z}$, is equal to the unity and (ii) the density of the particles is comparable to the density of water, $\rho_{w}$. As a result, we conclude that

$$
c_{n p}^{t p z}=\rho_{w} f^{t p z}
$$

and we compute the value of $C_{\max }^{n p}$ that is reported in Table III.

\subsection{Computational methods}

For complex geometrical configurations explicit solutions of problems (2), (11) and (18) are not available. Numerical simulations are the only way of applying the model to real cases. The discretization of the flow problem (2) is described in [16], and it is achieved by means of the finite element method that arises from the variational formulation of the problem combined with a partition of the domain into small elements. We follow the same method also to discretize problems (11) and (18). More precisely, starting from the problems of oxygen and TPZ mass transport, we multiply (11a) and (11b) for a test function $q_{t} \in V_{t}=H_{\alpha}^{1}(\Omega)$, where $H_{\alpha}^{1}(\Omega)$ with $\alpha \in(0,1)$ is the natural trial space for the problem in the interstitium, as discussed in [16] and references therein. We integrate over $\Omega$ and the transport operator is treated using integration by parts combined, for the sake of simplicity, with homogeneous Robin conditions (18b) on $\partial \Omega$ (Neumann conditions will be also addressed in the simulations). Regarding the interface flux term, we write

$$
\left\langle f_{c}^{*}\left(\bar{p}_{t}, p_{v}, \bar{c}_{t}^{*}, c_{v}^{*}\right) \delta_{\Lambda}, q_{t}\right\rangle_{\Omega}=\left\langle f_{c}^{*}\left(\bar{p}_{t}, p_{v}, \bar{c}_{t}^{*}, c_{v}^{*}\right), q_{t}\right\rangle_{\Lambda},
$$

where $\langle\cdot, \cdot\rangle_{\Sigma}$ denotes the standard inner product in $L^{2}(\Sigma)$ and the generic set $\Sigma$ stands for either $\Omega$ or $\Lambda$. We proceed similarly for the governing equation on the capillary bed. Integrating by parts on each branch $\Lambda_{i}$ separately, it is possible to manipulate the resulting equations in order to naturally impose the mass conservation at each node of the network, see [16] for details. This property is satisfied provided that the test functions of the pressure field on the capillary bed are continuous on 
the entire network, namely $q_{v} \in C^{0}(\Lambda)$. In particular we choose $V_{v, 0}$ as the subspace of $H^{1}(\Lambda)$ of functions which vanish on the boundaries of $\Lambda$ and therefore $V_{v, 0} \subset C^{0}(\Lambda)$ on 1D manifolds. This allows us to obtain

$$
\left\langle\frac{\partial c_{v}^{*}}{\partial t}, q_{v}\right\rangle_{\Lambda}+\left\langle\left(\mathbf{u}_{v} \cdot \lambda\right) c_{v}^{*}-D_{v}^{*} \frac{\partial c_{v}^{*}}{\partial s}, \frac{\partial q_{v}}{\partial s}\right\rangle_{\Lambda}=\left\langle-\frac{1}{\pi R^{2}} f_{c}^{*}\left(\bar{p}_{t}, p_{v}, \bar{c}_{t}^{*}, c_{v}^{*}\right), q_{v}\right\rangle_{\Lambda}, \forall q_{v} \in V_{v, 0}
$$

Then, the weak formulation of (11) requires to find $c_{t}^{*} \in V_{t}$ and $c_{v}^{*} \in V_{v, 0}$ with $*=o x, t p z$ such that,

$$
\left\{\begin{array}{l}
\left\langle\frac{\partial c_{t}^{*}}{\partial t}, q_{t}\right\rangle_{\Omega}+a_{t}\left(c_{t}^{*}, q_{t}\right)-b_{\Lambda}^{t}\left(\bar{c}_{t}^{*}, q_{t}\right)=b_{\Lambda}^{t}\left(c_{v}^{*}, q_{t}\right), \forall q_{t} \in V_{t}, \\
\left\langle\frac{\partial c_{v}^{*}}{\partial t}, q_{v}\right\rangle_{\Lambda}+a_{v}\left(c_{v}^{*}, q_{v}\right)-b_{\Lambda}^{v}\left(c_{v}^{*}, q_{v}\right)=b_{\Lambda}^{v}\left(\bar{c}_{t}^{*}, q_{c}\right), \forall q_{c} \in V_{v, 0},
\end{array}\right.
$$

with the following bilinear forms,

$$
\begin{aligned}
a_{t}\left(c_{t}^{*}, q_{t}\right) & :=\left\langle c_{t}^{*} \mathbf{u}_{t}-D_{t}^{*} \nabla c_{t}^{*}, \nabla q_{t}\right\rangle_{\Omega}+\left\langle m^{*}\left(c_{t}^{o x}\right) c_{t}^{*}, q_{t}\right\rangle_{\Omega}+L_{p}^{L F} \frac{S}{V}\left\langle\left(p_{t}-p_{L}\right) c_{t}^{*}, q_{t}\right\rangle_{\Omega}, \\
a_{v}\left(c_{v}^{*}, q_{v}\right) & :=\left\langle\left(\mathbf{u}_{v} \cdot \lambda\right) c_{v}^{*}-D_{v}^{*} \frac{\partial c_{v}^{*}}{\partial s}, \frac{\partial q_{v}}{\partial s}\right\rangle_{\Lambda}, \\
b_{\Lambda}^{t}\left(c_{v}^{*}, q_{c}\right) & :=\left\langle 2 \pi R\left[\left(1-\sigma^{*}\right) L_{p}\left(\left(p_{v}-\bar{p}_{t}\right)-\sigma^{p}\left(\pi_{v}^{p}-\bar{\pi}_{t}^{p}\right)\right)\left(1-w^{*}\right) c_{v}^{*}+P^{*} c_{v}^{*}\right], q_{c}\right\rangle_{\Lambda}, \\
b_{\Lambda}^{t}\left(\bar{c}_{t}^{*}, q_{t}\right) & :=\left\langle 2 \pi R\left[\left(1-\sigma^{*}\right) L_{p}\left(\left(p_{v}-\bar{p}_{t}\right)-\sigma^{p}\left(\pi_{v}^{p}-\bar{\pi}_{t}^{p}\right)\right) w^{*} \bar{c}_{t}^{*}-P^{*} \bar{c}_{t}^{*}\right], q_{t}\right\rangle_{\Lambda}, \\
b_{\Lambda}^{v}\left(c_{v}^{*}, q_{c}\right) & :=\left\langle 2 / R\left[\left(1-\sigma^{*}\right) L_{p}\left(\left(p_{v}-\bar{p}_{t}\right)-\sigma^{p}\left(\pi_{v}^{p}-\bar{\pi}_{t}^{p}\right)\right)\left(1-w^{*}\right) c_{v}^{*}+P^{*} c_{v}^{*}\right], q_{c}\right\rangle_{\Lambda}, \\
b_{\Lambda}^{v}\left(\bar{c}_{t}^{*}, q_{t}\right) & :=\left\langle 2 / R\left[\left(1-\sigma^{*}\right) L_{p}\left(\left(p_{v}-\bar{p}_{t}\right)-\sigma^{p}\left(\pi_{v}^{p}-\bar{\pi}_{t}^{p}\right)\right) w^{*} \bar{c}_{t}^{*}-P^{*} \bar{c}_{t}^{*}\right], q_{t}\right\rangle_{\Lambda} .
\end{aligned}
$$

We proceed in a similar way also for (18). The variational problem for nanoparticle transport and TPZ delivery requires to find $c_{t}^{t p z} \in V_{t}$ and $c_{v} \in V_{v, 0}$ such that

$$
\left\{\begin{array}{l}
\left\langle\frac{\partial c_{t}^{t p z}}{\partial t}, q_{t}\right\rangle_{\Omega}+a_{t}^{t p z}\left(c_{t}^{t p z}, q_{t}\right)=F(t), \forall q_{t} \in V_{t}, \\
\left\langle\frac{\partial c_{v}}{\partial t}, q_{v}\right\rangle_{\Lambda}+a_{v}^{t p z}\left(c_{v}, q_{v}\right)=0, \forall q_{c} \in V_{v, 0}
\end{array}\right.
$$

and the bilinear forms are

$$
\begin{aligned}
& a_{t}^{t p z}\left(c_{t}^{t p z}, q_{t}\right):=\langle\left.c_{t}^{t p z} \mathbf{u}_{t}-D_{t}^{t p z} \nabla c_{t}^{t p z}, \nabla q_{t}\right\rangle_{\Omega}+\left\langle m^{t p z}\left(c_{t}^{o x}\right) c_{t}^{t p z}, q_{t}\right\rangle_{\Omega} \\
&+\left\langle F^{L F} p \frac{S}{V}\left(p_{t}-p_{L}\right) c_{t}^{t p z}, q_{t}\right\rangle_{\Omega}, \\
& a_{v}^{t p z}\left(c_{v}, q_{v}\right):=\left\langle\left(\mathbf{u}_{v} \cdot \lambda\right) c_{v}-D_{v} \frac{\partial c_{v}}{\partial s}, \frac{\partial q_{v}}{\partial s}\right\rangle_{\Lambda}+\left\langle\frac{2 \pi R}{\pi R^{2}} \Pi c_{v}, q-v\right\rangle_{\Lambda}, \\
& F(t):=\left\langle 2 \pi R J(t), q_{t}\right\rangle_{\Lambda} .
\end{aligned}
$$

For the spatial approximation, we first introduce an admissible family of partitions of $\Omega$ into tetrahedrons $K \in \mathcal{T}_{t}^{h}$, where the apex $h$ denotes the mesh characteristic size. For the discretization of the capillary bed, each branch $\Lambda_{i}$ is partitioned into a sufficiently large number of linear segments $E$, whose collection is $\Lambda_{i}^{h}$, which represents a finite element mesh on a one-dimensional manifold. Let $\Lambda^{h}:=\cup_{i=1}^{N} \Lambda_{i}^{h}$ be the finite element partition of the entire capillary bed. At the discrete level, one of the advantages of our problem formulation is that the partition of the domains $\Omega$ and $\Lambda$ into elements are completely independent. The computational meshes used to solve the transport problems are reported in Figure 2. Let $V_{t}^{h}:=\left\{v \in C^{0}(\Omega):\left.v\right|_{K} \in \mathbb{P}^{1}(K), \forall K \in \mathcal{T}_{t}^{h}\right\}$ be the space of piecewise 

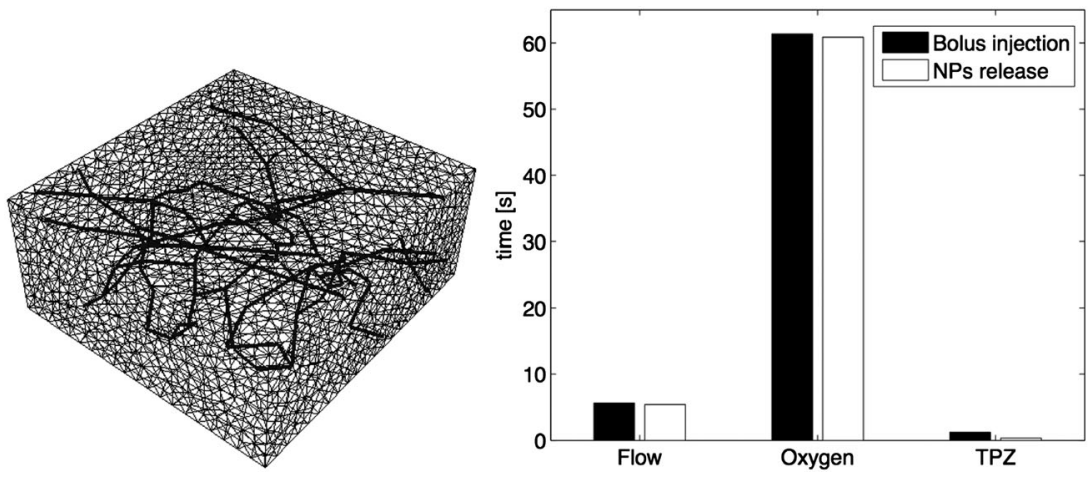

Figure 2. On the left: meshes used to solve problems (23), (24) and (25). The partition of the domains $\Omega$ and $\Lambda$ into elements are completely independent. In particular, the partition of $\Omega$ is composed by 32,624 elements, while the partition of $\Lambda$ is composed by 8400 nodes, 80 nodes for each branch. On the right: computational time for solving the algebraic systems of the flow equations, the oxygen transport problem and the TPZ mass transport problem for the two different modalities of delivery. We represent one single time step for the solution of the TPZ transport problem. The bars quantify the CPU time measured in seconds.

linear continuous finite elements on $\mathcal{T}_{t}^{h}$ and let $V_{v, i}^{h}:=\left\{v \in C^{0}\left(\Lambda_{i}\right):\left.v\right|_{E} \in \mathbb{P}^{1}(E), \forall E \in \Lambda_{i}^{h}\right\}$ be the piecewise linear and continuous finite element space on $\Lambda_{i}$. The numerical approximation of the equation posed on the capillary bed is then achieved using the space $V_{v}^{h}:=\left(\cup_{i=1}^{N} V_{v, i}^{h}\right) \cap$ $C^{0}(\Lambda)$. The discrete problems arising from (20) and (21) require to find $c_{t}^{*, h} \in V_{t}^{h}, c_{v}^{*, h} \in V_{v, 0}^{h}$ and $c_{t}^{t p z, h} \in V_{t}^{h}$ and $c_{v}^{h} \in V_{v, 0}^{h}$ such that

$$
\begin{gathered}
\begin{cases}\left\langle\frac{\partial c_{t}^{*, h}}{\partial t}, q_{t}^{h}\right\rangle_{\Omega}+a_{t}\left(c_{t}^{*, h}, q_{t}^{h}\right)-b_{\Lambda^{h}}^{t}\left(\bar{c}_{t}^{*, h}, q_{t}^{h}\right)=b_{\Lambda^{h}}^{t}\left(c_{v}^{*, h}, q_{t}^{h}\right), \forall q_{t}^{h} \in V_{t}^{h}, \\
\left\langle\frac{\partial c_{v}^{*, h}}{\partial t}, q_{v}^{h}\right\rangle_{\Lambda}+a_{v}\left(c_{v}^{*, h}, q_{v}^{h}\right)-b_{\Lambda^{h}}^{v}\left(c_{v}^{*, h}, q_{v}^{h}\right)=b_{\Lambda^{h}}^{v}\left(\bar{c}_{t}^{*, h}, q_{v}^{h}\right), \forall q_{v}^{h} \in V_{v, 0}^{h},\end{cases} \\
\begin{cases}\left\langle\frac{\partial c_{t}^{t p z, h}}{\partial t}, q_{t}^{h}\right\rangle_{\Omega}+a_{t}^{t p z}\left(c_{t}^{t p z, h}, q_{t}^{h}\right)=F(t), & \forall q_{t}^{h} \in V_{t}^{h}, \\
\left\langle\frac{\partial c_{v}^{h}}{\partial t}, q_{v}^{h}\right\rangle_{\Lambda}+a_{v}^{t p z}\left(c_{v}^{h}, q_{v}^{h}\right)=0, & \forall q_{v}^{h} \in V_{v, 0}^{h},\end{cases}
\end{gathered}
$$

where the bilinear forms $a_{t}(\cdot, \cdot), a_{v}(\cdot, \cdot), b^{\Lambda^{h}}(\cdot, \cdot), a_{t}^{t p z}(\cdot, \cdot), a_{v}^{t p z}(\cdot, \cdot)$ are the same as before, with the only difference that $b^{\Lambda^{h}}(\cdot, \cdot)$ is now defined over the discrete representation of the network $\Lambda_{h}$. The interpolation and average operators, which are needed to evaluate the bilinear form $b^{\Lambda^{h}}(\cdot, \cdot)$, are described in [16], and the error analysis of the present scheme can be addressed with the tools provided in [20].

The space discretization must be complemented with a time advancing scheme. For the numerical approximation of the variational problems (22) and (23), we consider a standard backward Euler time advancing method. Let $\Delta t>0$ be the time step, $t_{n}=n \Delta t$ the $n$-th time point, and $c_{t}^{*, h, n} \in$ $V_{t}^{h}, c_{v}^{*, h, n} \in V_{v, 0}^{h}$, the numerical approximations of $c_{t}^{*, h}\left(t_{n}\right)$ and $c_{v}^{*, h}\left(t_{n}\right)$. As a result, we obtain the following discrete problems: given $c_{t}^{*, h, n} \in V_{t}^{h}$ and $c_{v}^{*, h, n} \in V_{v, 0}^{h}$ find $c_{t}^{*, h, n+1} \in V_{t}^{h}$ and $c_{v}^{*, h, n+1} \in V_{v, 0}^{h}$, such that 


$$
\left\{\begin{array}{c}
\left\langle\frac{1}{\Delta t} c_{t}^{*, h, n+1}, q_{t}^{h}\right\rangle_{\Omega}+a_{t}\left(c_{t}^{*, h, n+1}, q_{t}^{h}\right)-b_{\Lambda^{h}}^{t}\left(c_{t}^{-*, h, n+1}, q_{t}^{h}\right) \\
=\left\langle\frac{1}{\Delta t} c_{t}^{*, h, n}, q_{t}^{h}\right\rangle_{\Omega}+b_{\Lambda^{h}}^{t}\left(c_{v}^{*, h, n+1}, q_{t}^{h}\right), \forall q_{t}^{h} \in V_{t}^{h} \\
\left\langle\frac{1}{\Delta t} c_{v}^{*, h, n+1}, q_{v}^{h}\right\rangle_{\Lambda}+a_{v}\left(c_{v}^{*, h, n+1}, q_{v}^{h}\right)-b_{\Lambda^{h}}^{v}\left(c_{v}^{*, h, n+1}, q_{v}^{h}\right) \\
=\left\langle\frac{1}{\Delta t} c_{v}^{*, h, n}, q_{v}^{h}\right\rangle_{\Lambda}+b_{\Lambda^{h}}^{v}\left(c_{t}^{-*, h, n+1}, q_{v}^{h}\right), \forall q_{v}^{h} \in V_{v, 0}^{h} .
\end{array}\right.
$$

An equivalent approach is applied to discretize (23).

Finally, we observe that the equation which describes the oxygen concentration transport, (11), involves a nonlinear term, represented by the Michelis-Menten reaction formula. To solve the problem, we apply an iterative scheme strategy, where the oxygen concentration is evaluated at the previous iterative step. For simplicity of notation, to address this iterative scheme, we drop the time index $n+1$. This index will be explicitly indicated only when referring to a time step different than $t_{n+1}$. For the same reason, we drop the index $h$ everywhere. Then, for all $n=1, \ldots, N$ given an initial guess $c_{t}^{o x, 0}, c_{v}^{o x, 0}$ and a tolerance $\varepsilon$, the iterative strategy consists to find a sequence $c_{t}^{o x, k}, c_{v}^{o x, k}$ for $k=1,2, \ldots$ such that,

$$
\left\{\begin{aligned}
&\left\langle\frac{1}{\Delta t} c_{t}^{o x, k}, q_{t}\right\rangle_{\Omega}+\left\langle c_{t}^{o x, k} \mathbf{u}_{t}-D_{t}^{o x} \nabla c_{t}^{o x, k}, \nabla q_{t}\right\rangle_{\Omega} \\
&+\left\langle m^{o x}\left(c_{t}^{o x, k-1}\right) c_{t}^{o x, k}, q_{t}\right\rangle_{\Omega}+L_{p}^{L F} \frac{S}{V}\left\langle\left(p_{t}-p_{L}\right) c_{t}^{o x, k}, q_{t}\right\rangle_{\Omega} \\
&=\left\langle\frac{1}{\Delta t} c_{t}^{o x, k, n}, q_{t}\right\rangle_{\Omega}+b_{\Lambda^{h}}^{t}\left(\bar{c}_{t}^{o x, k}, q_{t}\right)+b_{\Lambda^{h}}^{t}\left(c_{v}^{o x, k}, q_{t}\right), \forall q_{t} \in V_{t}^{h} \\
&\left\langle\frac{1}{\Delta t} c_{v}^{o x, k}, q_{v}\right\rangle_{\Lambda}+a_{v}\left(c_{v}^{o x, k}, q_{v}\right)-b_{\Lambda^{h}}^{v}\left(c_{v}^{o x, k}, q_{v}\right) \\
&=\left\langle\frac{1}{\Delta t} c_{v}^{o x, k, n}, q_{v}\right\rangle_{\Lambda}+b_{\Lambda^{h}}^{v}\left(\bar{c}_{t}^{o x, k}, q_{v}\right), \forall q_{v} \in V_{v, 0}^{h}
\end{aligned}\right.
$$

until the following stopping criterion is satisfied:

$$
\frac{\left\|c_{t}^{o x, k}-c_{t}^{o x, k-1}\right\|_{0}}{\left\|c_{t}^{o x, k}\right\|_{0}}+\frac{\left\|c_{v}^{o x, k}-c_{v}^{o x, k-1}\right\|_{0}}{\left\|c_{v}^{o x, k}\right\|_{0}}<\varepsilon,
$$

where $\|\cdot\|_{0}$ is the Euclidean norm of the vector of nodal values.

Regarding the coupling between the oxygen and the TPZ concentration, we actually solve the steady counterpart of (24) for the oxygen transport, because oxygen is persistently supplied by the capillary bed. Therefore, once computed the oxygen concentration profile, we use it to determine once for all the reaction term that appears in the TPZ transport equation. This choice seems to be reasonable also because there isn't any feedback of the TPZ concentration on the oxygen consumption.

For the numerical solution of problems (25), (24) and (23), we use GetFem++, a general purpose $\mathrm{C}++$ finite element library [38]. The discretization of flow problem (2), already described in [16], is solved applying the GMRES method with incomplete-LU preconditioning. The tolerance for the stopping criterion for the iterative method to solve the oxygen transport (25) is fixed to $\varepsilon=10^{-8}$. We reach the convergence in 61 iterations. At each iteration, we apply the GMRES method to solve the corresponding linear systems. Regarding the TPZ transport, the monolithic algebraic system constructed from (24) is again solved using the GMRES method with incomplete-LU preconditioning. Conversely, the two equations composing system (23) are actually decoupled, therefore they are addressed in sequence: we solve the vessel (23b) first, in order to compute the flux $J(t)$, which is 
Table IV. Computational time for solving different parts of problems (23), (24) and (25). Computational time is measured in seconds.

\begin{tabular}{lcc}
\hline \multicolumn{1}{c}{ Task } & Bolus injection & Nanoparticle release \\
\hline Problems initialization & 301.7 & 301.59 \\
Assembling fluid system & 1.02 & 1.04 \\
Solving fluid system & 5.62 & 5.39 \\
Assembling $O_{2}$ system & 1.32 & 1.35 \\
Solving $O_{2}$ system & 61.36 & 60.86 \\
Assembling drug system & 1.37 & 1.48 \\
Solving drug system (one single step) & 1.2 & 0.21 \\
Solving drug system $(T=20$ min $)$ & 3374.04 & 1231.24 \\
\hline
\end{tabular}

the forcing term of the tissue (23a). Because these equations are independent, their numerical solution turns out to be faster than the one of system (24), as we observe from the results reported in Figure 2 and in Table IV.

Examining the coefficients of Table II, we observe that in (11) reaction terms dominate in the interstitial tissue. For this reason, we adopt the mass lumping stabilization techniques addressed in [39], while numerical experiments confirm that resorting to stabilization methods for the convective terms is not required for the applications that will be addressed here.

\section{RESULTS AND DISCUSSION}

The delivery of anticancer agents mediated through nanoparticle injection in the blood stream features significant advantages with respect to the traditional drug bolus delivery, because it increases the permanence of drug available in the systemic circulation [32]. We aim to explore the potential of the proposed simulation framework to capture these effects.

\subsection{Indicators of drug delivery performance}

The concentration profiles in the vessels, $c_{v}(t, s)$, and in the tissue, $c_{t}(t, x)$ are the natural outputs of the mass transport model described so far. From the clinical standpoint, these may not be the most significant indicators of the treatment performance. For this reason, we also study the amount of TPZ metabolized by cells up to a given reference time. We denote this quantity as $M^{t p z}(t, x)$. In addition, for more quantitative comparisons, we look at the total amount of TPZ metabolized in the considered portion of tissue, that is $\bar{M}^{t p z}(t)$. On the basis of the Michaelis-Menten metabolization kinetics adopted in (11), these indicators are defined as

$$
M^{t p z}(t, \boldsymbol{x})=\int_{0}^{t} m^{t p z}\left(c_{t}^{o x}(\tau, \boldsymbol{x}), c_{t}^{t p z}(\tau, \boldsymbol{x})\right) d \tau, \quad \bar{M}^{t p z}(t)=\int_{\Omega} M^{t p z}(t, \boldsymbol{x}) d \boldsymbol{x} .
$$

Following [2], the amount of drug metabolized in the tissue can be related to the cell survival. In particular, the cell surviving fraction $(S F)$ represents the complement of the fraction of cells treated (killed) by TPZ with respect to the number of control cells (the total number of cells in the tissue, before treatment started). Several models are available to quantify the surviving fraction [2]. In particular, we use

$$
S F(t, \boldsymbol{x})=\exp \left(-\alpha M^{t p z}(t, \boldsymbol{x})\right),
$$

where $\alpha$ is a phenomenological coefficient. For the following calculations we assume $\alpha=2.52 \times$ $10^{-4} \mu \mathrm{M}^{-1}\left(0.0014 \mathrm{~g} / \mathrm{m}^{3}\right)$ as in [2].

\subsection{Oxygen transport and TPZ delivery from bolus injection}

We analyze the simulations of oxygen and TPZ transport obtained with model (11). In Figure 3, we compare the oxygen and the TPZ concentrations $20 \mathrm{~min}$ after that the delivery of TPZ into the 

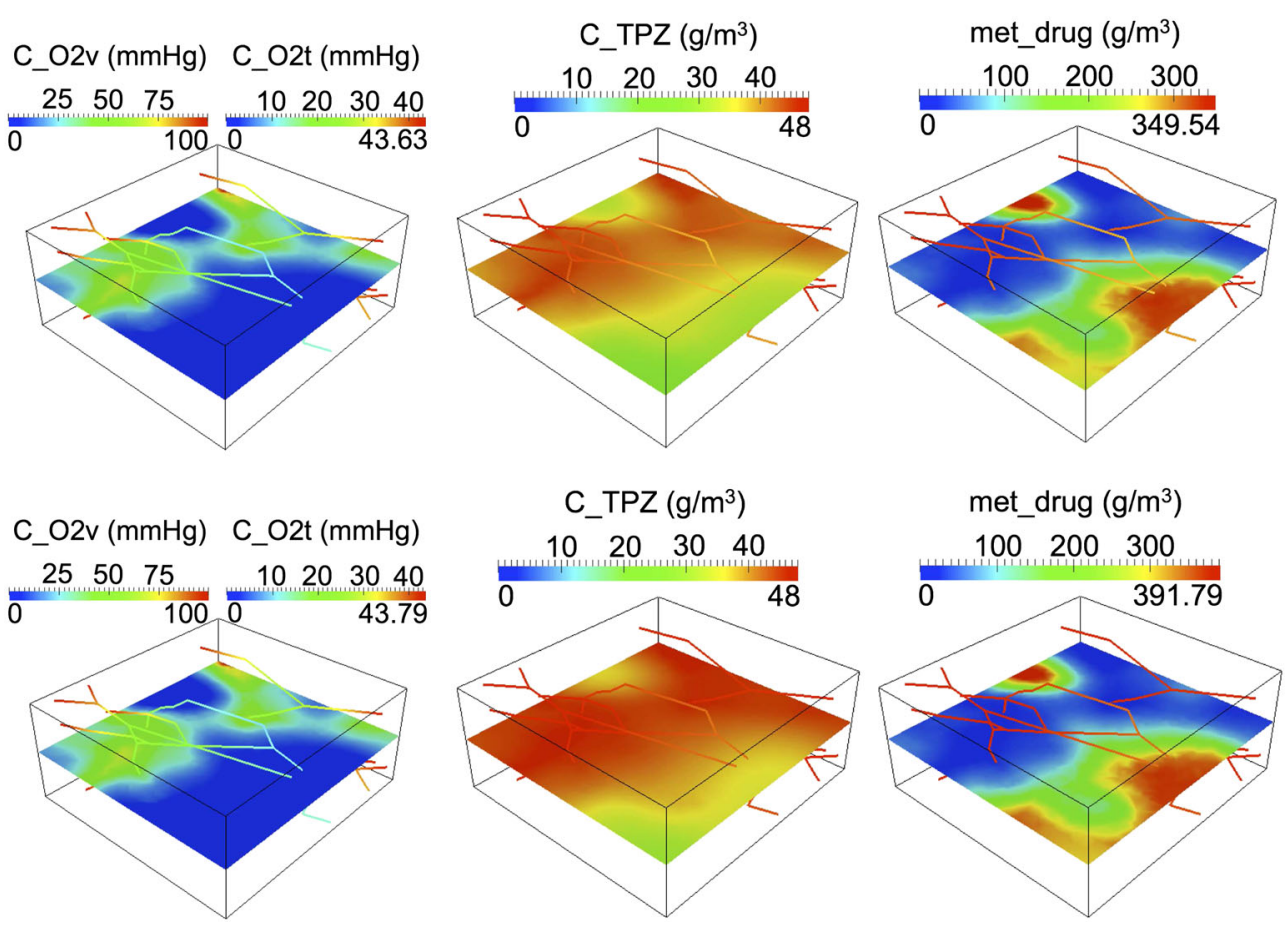

Figure 3. Oxygen concentration profile, bolus delivered TPZ concentration profile and metabolized drug profile are visualized from left to right. On the top row, simulations are performed using Robin boundary conditions for the concentrations of oxygen and TPZ at the boundary of the interstitial volume with the exterior. The results obtained using homogeneous Neumann conditions are depicted below.

systemic circulation has started. Oxygen concentration pattern substantially depends on the density of capillaries per unit volume. Regions of the sample tissue not well perfused by the capillary network show low oxygen concentrations, justifying the risk of hypoxic conditions for an irregular configuration of the microvessels. This conclusion is also supported by the dimensional analysis of the governing equations. Because oxygen transport in the interstitial volume is reaction dominated, regions free of oxygen sources will easily experience low oxygen supply. The visualization of oxygen concentration maps of Figure 3 can be directly compared with the results of [17], see in particular the top row of Figure 3, obtained using an equivalent model for oxygen transport. As a preliminary and qualitative validation of our results, we observe that the contour plots of the calculated oxygen concentration look remarkably similar in the two cases. As expected, the TPZ concentration is significantly influenced by the distribution of oxygen concentration. The distribution of TPZ in the considered tissue sample seems to be more uniform than in the case of oxygen.

In spite of the difference between the governing mechanisms at the basis of oxygen and TPZ transport, the simulated concentration maps of these species share common traits. This may be explained by two concurrent factors. On one hand, both solutes are affected by the distribution of capillaries. On the other hand, the metabolization of TPZ increases in hypoxic regions. This effect sustains TPZ concentration gradients similar to the ones of oxygen, by turning off TPZ absorption where oxygen concentration is elevated, and promoting TPZ metabolization where oxygen is low. Finally, the inspection of metabolized TPZ, namely $M^{t p z}(t, x)$, shows that the objective of reaching the hypoxic regions with a chemotherapy agent is substantially achieved, see Figure 3 (left). More precisely, oxygen and $M^{t p z}(t, x)$ maps show a complementary pattern. It means that most of TPZ is metabolized in hypoxic regions.

Before proceeding, we study the sensitivity of these results with respect to the boundary conditions applied on the artificial sections separating the interstitial volume from the exterior. The simulations reported in Figure 3 (top row) are obtained using $-D_{t} \nabla c_{t} \cdot \mathbf{n}=\beta_{c} c_{t}$ where the value of $\beta_{c}$ is reported in Table II. We compare these results with Figure 3 (bottom row), showing the 
concentration maps when homogeneous Neumann conditions (no flux) are prescribed for the concentrations on the boundary of $\Omega$. This is equivalent to set $\beta_{c}=0$. A slight increase in the TPZ concentration field is observed, in agreement with the fact that the outgoing diffusive flux is set to zero with the choice $\beta_{c}=0$. For a more quantitative comparison, we study the sensitivity of the total amount of metabolized TPZ, $\bar{M}^{t p z}(t)$. After injecting TPZ for 20 min, we calculate $\bar{M}^{t p z}(t)=$ $7.76838 \times 10^{-9} \mathrm{~g} / \mathrm{m}^{3}$ when using Robin boundary conditions and $\bar{M}^{t p z}(t)=8.6685 \times 10^{-9} \mathrm{~g} / \mathrm{m}^{3}$ in the case of Neumann conditions. Owing to these results, we conclude that the parameter $\beta_{c}$ is not a factor of primary importance to determine the concentrations of oxygen and TPZ and we will use Robin conditions for all the forthcoming simulations.

\subsection{Nanoparticle adhesion patterns and delivery of TPZ from nanoparticle injection}

We split the analysis of the TPZ delivery from nanoparticles in two parts. First, we focus on the nanoparticle adhesion model, with the aim to validate our results with respect to the ones reported in [34]. In a second phase, we analyze the concentration of TPZ delivered from the nanoparticles that decorate the capillary walls.

Nanoparticle adhesion is regulated by the vascular adhesion parameter $\Pi$, which in turn depends on the shear rate arising from the interaction of blood flow with the capillary walls. These two quantities are depicted in Figure 4. We observe that the wall shear rate features a significant spatial variation, although the capillary radius is constant along the network. This effect is due to the variable pressure, and consequently variable flow rate, along the network. We observe that the calculated values of wall shear rate fall in the physiological range [34]. According to the adopted adhesion model, the variability of wall shear rate is propagated to the adhesion parameter, reported
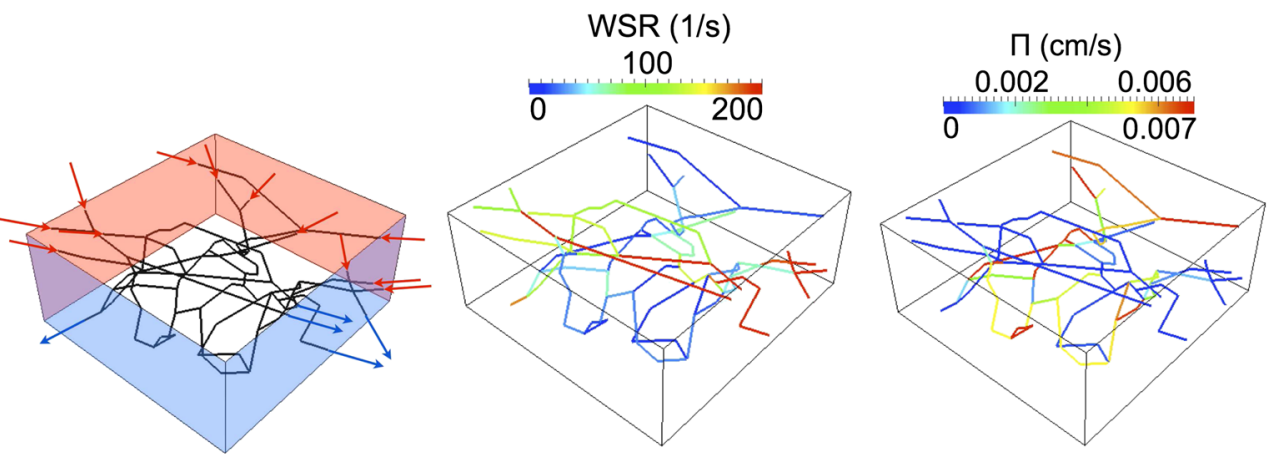

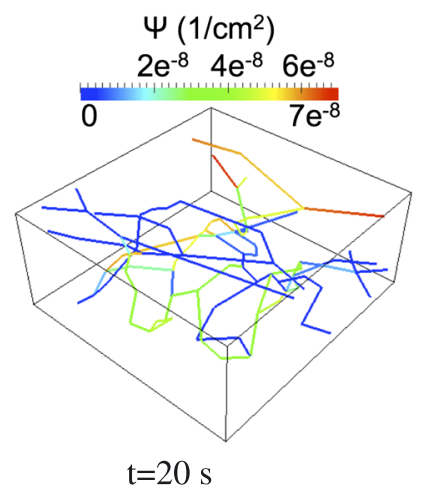

normalized $C_{\max }^{n p}$

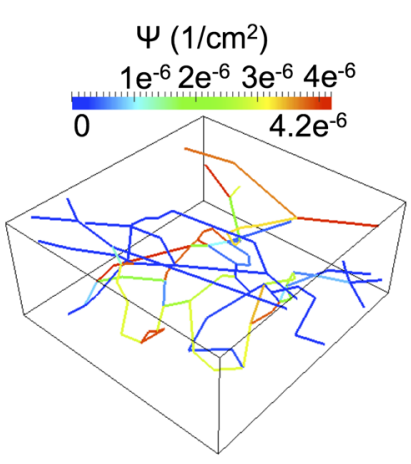

$\mathrm{t}=20 \min$

normalized $C_{m a x}^{n p}$

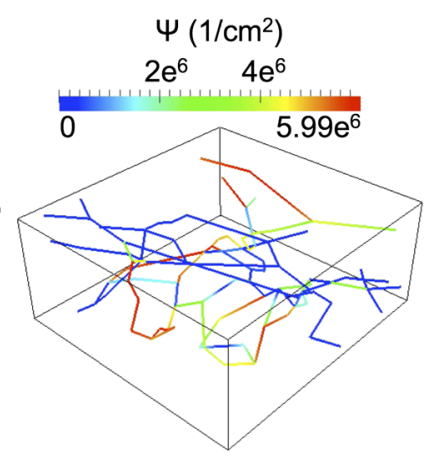

$\mathrm{t}=20 \min$

absolute $C_{\max }^{n p}$

Figure 4. Top panel: Capillary network profiles of wall shear rate (WSR) and vascular deposition parameter $\Pi$. Bottom, starting from the left: the density of nanoparticles decorating the wall normalized to the injected value, $\Psi$, at $20 \mathrm{~s}$ and at $20 \mathrm{~min}$ after nanoparticle injection has started. These values refer to a nominal unit concentration of injected particles $\left(\sharp / \mathrm{m}^{3}\right)$. Bottom right: $\Psi$ at $20 \mathrm{~min}$ after injection for an inlet nanoparticle concentration equal to $C_{\max }^{n p}$. 

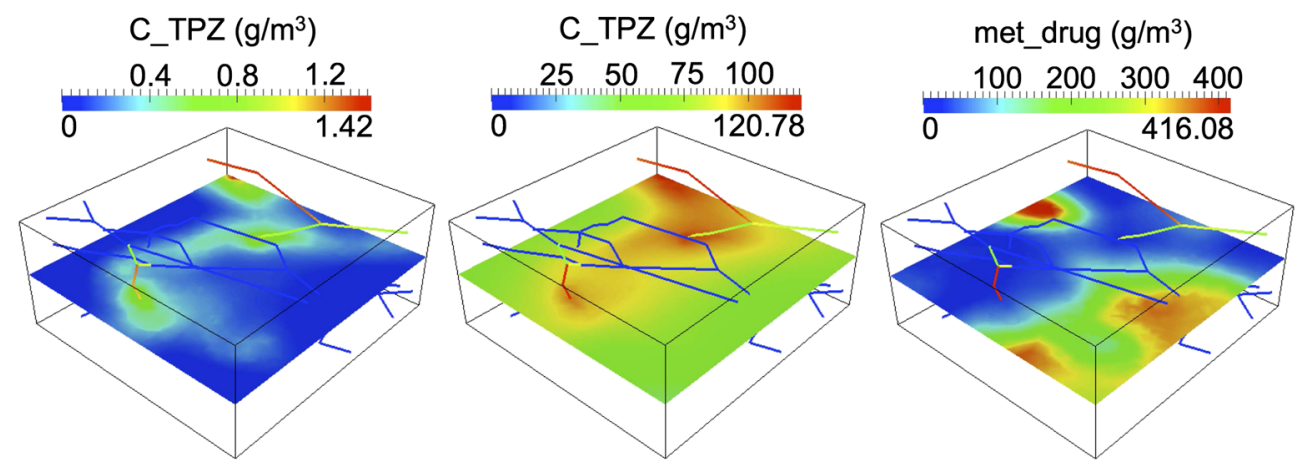

Figure 5. TPZ concentration maps at $20 \mathrm{~s}$ and $20 \mathrm{~min}$ after starting nanoparticle release (left and central panels). On the right, we show the metabolized drug after $20 \mathrm{~min}$. Robin boundary conditions are used in all cases.

on Figure 4 (left). As a result, we expect to observe a nonuniform concentration of nanoparticles adhering to the wall.

The nanoparticle density per unit capillary surface, namely $\Psi(s, t)$, depends on the adhesion parameter and on the particle concentration traveling through the vascular network. A preliminary validation of our simulations arises observing that the nanoparticle density per unit capillary surface, calculated for an injection phase lasting $20 \mathrm{~s}$, is comparable to the one reported in [34]. To compare the delivery of TPZ from nanoparticles with the case of bolus injection, we consider a constant concentration of injected particles for $20 \mathrm{~min}$. The analysis of adhered particles at $20 \mathrm{~min}$ after the initial time, shows that adhesion progressively increases. The results of Figure 4 (left and middle panels, bottom row) refer to the concentration of adhered nanoparticles normalized with respect to the injected value. On the right, we show the density of adhering particles when we consider the initial particle concentration $C_{\text {max }}^{n p}$, which is calculated in order to match the total flow of TPZ already used in the case of direct bolus injection.

Figure 5 shows the TPZ concentration delivered from nanoparticles at $20 \mathrm{~s}$ and 20 min after particle injection has started. Although the concentration levels are significantly different in the two cases, because of the time scales, the concentration maps share some similarities. In both cases, however, the geometry of the network cannot be immediately related to the TPZ concentration map. Indeed, it is rather the distribution of the adhesion factor along the network, $\Pi$, that affects the calculated concentration field. Finally, the amount of metabolized TPZ shown in Figure 5 (right) seems to be rather independent from the previous factors, but mostly influenced by the oxygen concentration field.

\subsection{Comparison of TPZ delivery from bolus and nanoparticle injection}

The proposed model enables us to compare how the concentration of delivered drug varies in space and time for the two considered modalities of drug delivery. We also point out that, although the delivery pathway is different, the comparisons refer to the same amount of drug injected into the system.

In Figure 6, we visualize the TPZ concentration maps in the two cases, reported at $20 \mathrm{~min}, 40 \mathrm{~min}$ and at a final times comparable to the time at which there will be no longer drug to be delivered. The magnitude of the end time may differ in bolus and nanoparticle injection. The first time point corresponds to the instant when the injection of drug or particles into the vessels is turned off. The analysis of the results reveals some differences since the beginning of the delivery process. The drug concentration in the case of nanoparticle delivery is larger than the one of bolus delivery at all time points and the discrepancy increases with time.

Figure 6 also shows that the concentration of TPZ delivered from bolus injection rapidly vanishes after the injection is switched off. At $40 \mathrm{~min}$ after the injection has started, there is only a negligible trace of TPZ in the tissue, while after $6 \mathrm{~h}$ the drug has completely vanished. This is clearly due to drug metabolization. Surprisingly, TPZ drug concentration from bolus delivery is also lower at 

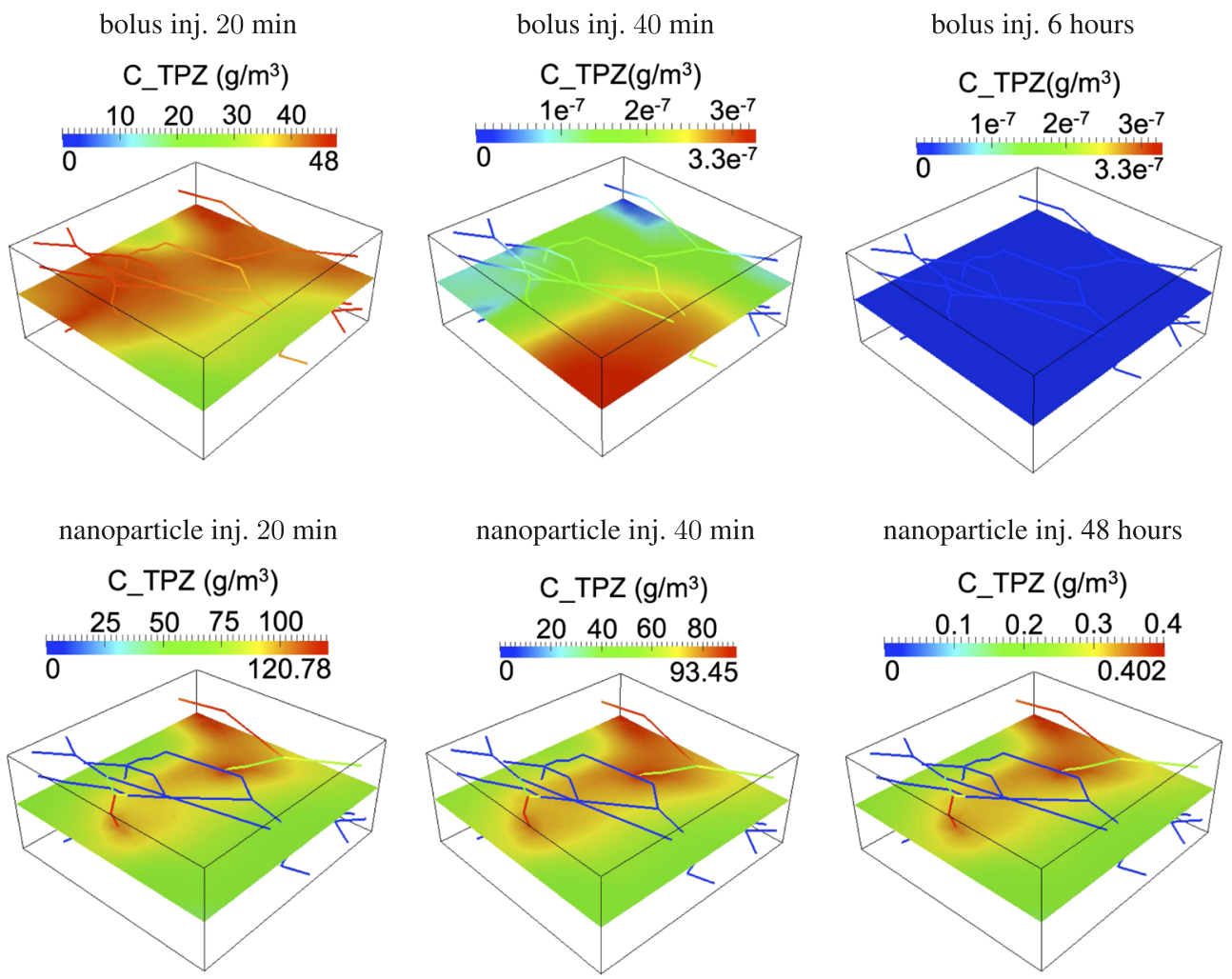

Figure 6. Comparison of TPZ concentrations released from bolus injection (top row) and nanoparticle injection (bottom row).

the first time point. The superior performance of the nanoparticle delivery system on the short time scale can be justified by the role of nanoparticle adhesion. This effect helps to harvest drug from the blood stream and to store it on the capillary walls. As a result, drug may be delivered in higher concentrations to the interstitial volume and at the same time a lower fraction of the injected drug is washed away by the blood stream leaving the tissue sample.

In addition, the release rate from nanoparticles is more persistent. Drug will be delivered to the tissue over a period of time that is significantly longer than $20 \mathrm{~min}$. This is due to the nanoparticle porous matrix, which represents a diffusional barrier to drug release. In this case, delivery and metabolization rates nearly balance, because the TPZ concentration in the interstitial volume slowly decreases for a period of almost $48 \mathrm{~h}$. This interpretation is supported by the visualization of the timecourse of the total drug amount available in the tissue, namely the volumetric integral of the TPZ concentration, reported in Figure 7. These results further highlight the inefficiency of drug bolus delivery when compared to drug delivery from nanoparticles.

The model suggests that bolus injection turns out to be a sub-optimal delivery strategy for two reasons. On one hand, tissue drug concentration rapidly reaches a plateau, much before the final injection time. The drug injected during this plateau phase is more likely to be washed out by the blood stream. On the other hand, the bolus injection system lacks of any buffer mechanism. Once the injection is switched off, drug levels rapidly decrease. In comparison, the nanoparticle delivery system features two significant advantages. First, the particle adhesion mechanism allows for the accumulation of drug on the capillary walls. Secondly, the presence of particles decorating the capillary walls ensures a persistent drug release rate after that the injection of particles has stopped.

The profiles of TPZ concentration have a direct impact on the amount of metabolized drug. The maps of metabolized drug are shown in Figure 8. We observe that these maps look alike in all reported cases. This similarity confirms the dominant role of oxygen concentration to selectively 

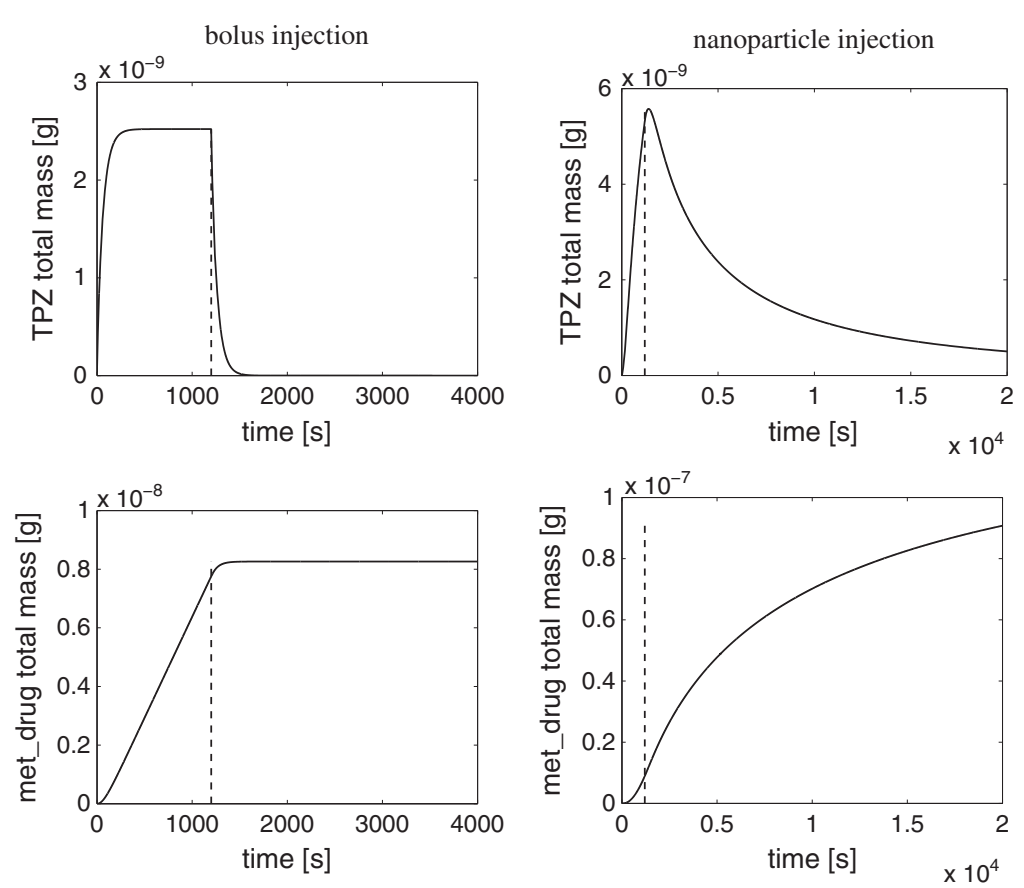

Figure 7. Comparison of systemic and nanoparticle release timecourses. The variation of $\int_{\Omega} c_{t}^{t p z}$ and $\bar{M}^{t p z}$ over time is visualized. The vertical dashed line marks the time at which the injection of drug or particles into the vessels is stopped.

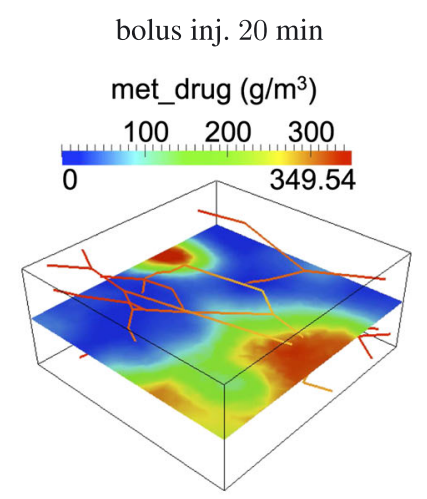

nanoparticle inj. $20 \mathrm{~min}$

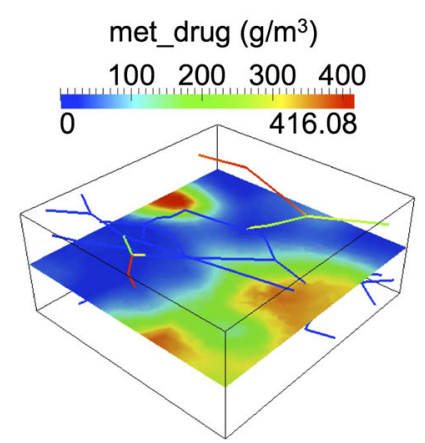

bolus inj. $40 \mathrm{~min}$

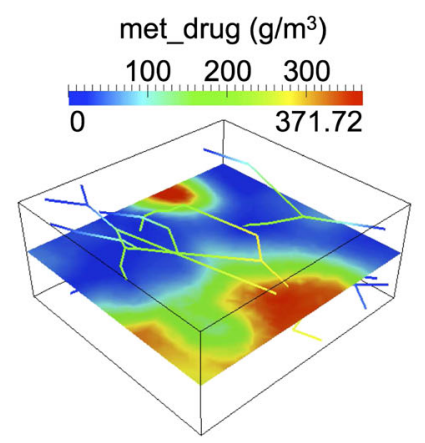

nanoparticle inj. $40 \mathrm{~min}$

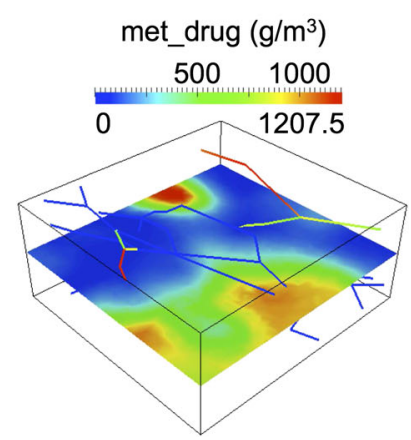

bolus inj. 6 hours

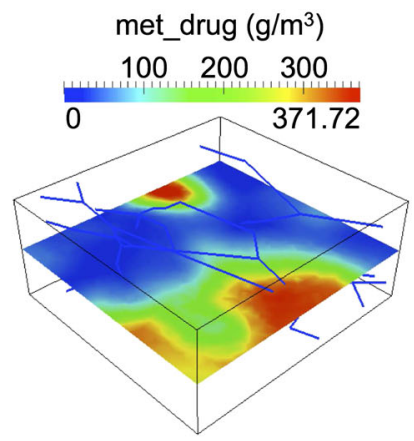

nanoparticle inj. 48 hours

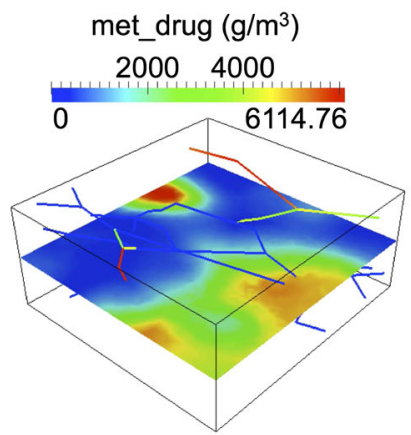

Figure 8. Comparison of metabolized TPZ released from bolus injection (top row) and nanoparticle injection (bottom row). 
activate the drug metabolization. However, the magnitude drastically changes from case to case. Because for the bolus delivery mode the drug supply to the tissue stops at $20 \mathrm{~min}$, the amount of metabolized drug remains almost constant after this time. In contrast, the buffer effect provided by the adhered nanoparticles is responsible to a significant increase of metabolized drug over time. More precisely, Figure 7 shows that $\bar{M}_{n p}^{t p z}$ is about 10 times larger than $\bar{M}_{b o l}^{t p z} 6 \mathrm{~h}$ after injection. Similarly, from Figure 8, we observe that the pointwise values of $M_{n p}^{t p z}$ and $M_{b o l}^{t p z}$ scale by a factor 20 at $48 \mathrm{~h}$ after delivery.

Finally, we compare the cell surviving fractions (SF) relative to bolus and nanoparticle injection. The cell surviving fraction depends on space and time, but also on the oxygen availability. To selectively attack tumor mass, TPZ it targeted to treat hypoxic tissue. As shown in [2], it is convenient to plot the dependence of SF on oxygen concentration. This visualization of SF is displayed in Figure 9. The points of the diagram correspond to the nodes of the computational grid in the interstitial volume, denoted with $\boldsymbol{x}_{i}$. For each node, we extract the value of oxygen concentration and surviving fraction, at the final time,

$$
\left(t=T, \boldsymbol{x}_{i}\right) \rightarrow c_{t}^{o x}\left(t=T, \boldsymbol{x}_{i}\right), S F\left(t=T, \boldsymbol{x}_{i}\right)
$$

Then, in the diagrams of Figure 9, the surviving fraction is plotted with respect to the corresponding oxygen concentration, while the spatial information is hidden. As expected, SF sharply decreases for low oxygen concentrations, confirming that TPZ is able to selectively target hypoxic regions. The nanoparticle delivery mode results to be more effective also with respect to this indicator. On a short time scale, equivalent to the injection time, the efficacy of the bolus injection treatment is not particularly satisfactory because more than $50 \%$ of the cells still survive in hypoxic regions. For nanoparticle injection, the plot of SF after 20 min has a similar pattern (Figure 9), but the minimum of SF reaches $10 \%$, confirming that this delivery modality is more effective. While the situation is almost unchanged for longer time scales in the case of bolus delivery, the performance further improves for nanoparticles on the time scale of $48 \mathrm{~h}$. Indeed, the SF profile has shifted downwards and its minimum reaches zero for low oxygen concentrations, meaning that almost all the cells in
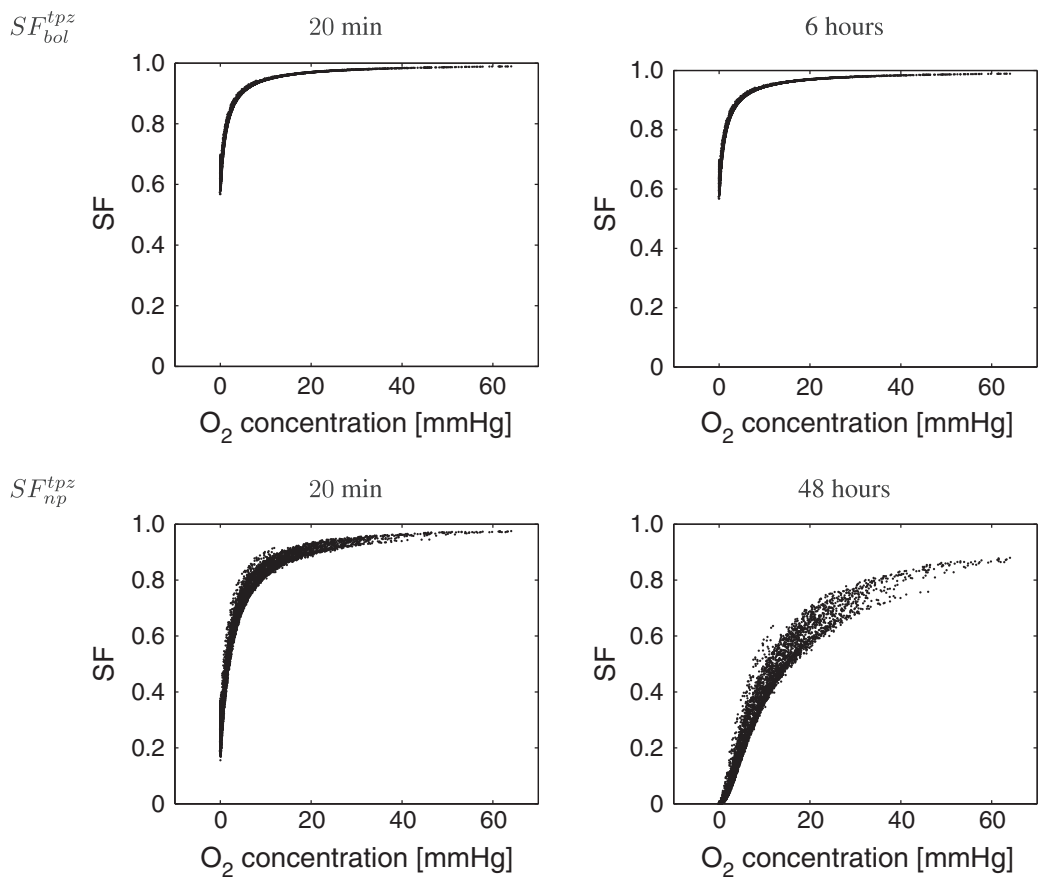

Figure 9. Comparison of cell surviving fraction (SF) when TPZ released from bolus injection (subscript $b o l$ ) and nanoparticle injection (subscript $n p$ ). 
the interstitial volume are treated. A slight drawback of the treatment based on nanoparticles can be detected looking at the distribution of the points in the SF plot (Figure 9). The dispersion of the point cloud increases and the slope of the underlying curve decreases with respect to the corresponding plot for bolus injection. This suggests that action of TPZ becomes less selective to target cells exposed to low oxygen concentration.

\section{CONCLUSIONS, LIMITATIONS AND FUTURE PERSPECTIVES}

In this study, we have developed a model capable to simulate the spatio-temporal evolution of drugs delivered to a tumor mass. The analysis is performed at the microscale, where the fundamental physics at the basis of flow and transport can be directly applied. We have used the model to compare bolus and nanoparticle injection for delivering chemotherapy agents. The model provides different insights on treatment performance, based on the analysis of specific quantitative indicators, such as the cell surviving fraction. The model suggests that bolus injection does not provide an optimal delivery. Drug washout by the blood stream and saturation of the concentration level in the interstitial tissue play as limiting factors for the amount of drug that reaches the interstitial volume, where malignant cells are active. The simulations confirm that a more controlled drug delivery process, achieved by means of nanoparticle injection, helps to override the previous limitations.

Besides these encouraging results, the model is open to several improvements. A current limitation is that it only approximately describes the complex phenomena involved in the interaction of red blood cells with fluid flow and oxygen transport. Although the full three-dimensional resolution of the fluid-particle interaction would be too demanding for our purposes, other reduced microcirculation models, such as [40,41], should be in future considered and eventually applied to this study. Another line of development consists in coping with the rapid technological progress in designing innovative methods to efficiently and selectively deliver drugs [31, 32]. Indeed, the model can be extended to encompass different drug delivery platforms. Further ramifications of this research will also be devoted to develop specific models for different types of cancer. We expect that tumors developing in the brain, breast, liver or lungs may feature significant differences in their transport properties. The physiology of these organs as well as available metrics to characterize their transport properties will be combined to set up specific variants of the model for different tumors. Another limitation of the study consists to consider a tumor as a static environment. Future developments of the model will indeed consider the tumor microenvironment as a dynamic system where angiogenesis, cell proliferation and drug treatment constantly interact.

\section{ACKNOWLEDGEMENT}

This work has been supported by the ERC Advanced Grant N.227058 MATHCARD.

\section{REFERENCES}

1. Hanahan D, Weinberg RA. The hallmarks of cancer. Cell 2000; 100(1):57-70.

2. Hicks KO, Pruijn FB, Secomb TW, Hay MP, Hsu R, Brown JM, Denny WA, Dewhirst MW, Wilson WR. Use of three-dimensional tissue cultures to model extravascular transport and predict in vivo activity of hypoxia-targeted anticancer drugs. Journal of the National Cancer Institute 2006; 98(16):1118-1128.

3. Carmeliet P, Jain RK. Angiogenesis in cancer and other diseases. Nature 2000; 407(6801):249-257.

4. Baxter LT, Jain RK. Transport of fluid and macromolecules in tumors. i. role of interstitial pressure and convection. Microvascular Research 1989; 37(1):77-104.

5. Baxter LT, Jain RK. Transport of fluid and macromolecules in tumors ii. role of heterogeneous perfusion and lymphatics. Microvascular Research 1990; 40(2):246-263.

6. Baxter LT, Jain RK. Transport of fluid and macromolecules in tumors. iii. role of binding and metabolism. Microvascular Research 1991; 41(1):5-23.

7. Baxter LT, Jain RK. Transport of fluid and macromolecules in tumors: Iv. a microscopic model of the perivascular distribution. Microvascular Research 1991; 41(2):252-272.

8. Chapelle D, Gerbeau JF, Sainte-Marie J, Vignon-Clementel IE. A poroelastic model valid in large strains with applications to perfusion in cardiac modeling. Computational Mechanics 2010; 46(1):91-101. 
9. Michler C, Cookson AN, Chabiniok R, Hyde E, Lee J, Sinclair M, Sochi T, Goyal A, Vigueras G, Nordsletten DA, Smith NP. A computationally efficient framework for the simulation of cardiac perfusion using a multi-compartment darcy porous-media flow model. International Journal for Numerical Methods in Biomedical Engineering 2013; 29(2):217-232.

10. Chapman SJ, Shipley RJ, Jawad R. Multiscale modeling of fluid transport in tumors. Bulletin of Mathematical Biology 2008; 70(8):2334-2357.

11. Shipley RJ, Chapman SJ. Multiscale modelling of fluid and drug transport in vascular tumours. Bulletin of Mathematical Biology 2010; 72(6):1464-1491.

12. Vilanova G, Colominas I, Gomez H. Capillary networks in tumor angiogenesis: From discrete endothelial cells to phase-field averaged descriptions via isogeometric analysis. International Journal for Numerical Methods in Biomedical Engineering 2013; 29(10):1015-1037.

13. Blake TR, Gross JF. Analysis of coupled intra- and extraluminal flows for single and multiple capillaries. Mathematical Biosciences 1982; 59(2):173-206.

14. Fleischman GJ, Secomb TW, Gross JF. The interaction of extravascular pressure fields and fluid exchange in capillary networks. Mathematical Biosciences 1986; 82(2):141-151.

15. Flieschman GJ, Secomb TW, Gross JF. Effect of extravascular pressure gradients on capillary fluid exchange. Mathematical Biosciences 1986; 81(2):145-164.

16. Cattaneo L, Zunino P. Computational models for fluid exchange between microcirculation and tissue interstitium. Networks and Heterogeneous Media 2014; 9(1):135-159.

17. Secomb TW, Hsu R, Park EYH, Dewhirst MW. Green's function methods for analysis of oxygen delivery to tissue by microvascular networks. Annals of Biomedical Engineering 2004; 32(11):1519-1529.

18. Sun Q, Wu GX. Coupled finite difference and boundary element methods for fluid flow through a vessel with multibranches in tumours. International Journal for Numerical Methods in Biomedical Engineering 2013; 29(3): $309-331$

19. D'Angelo C. Multiscale modeling of metabolism and transport phenomena in living tissues. Ph.D. Thesis, Ecole Polytechnique Federale de Lausanne (EPFL), Lausanne, Switzerland, 2007.

20. D'Angelo C. Finite element approximation of elliptic problems with dirac measure terms in weighted spaces: applications to one- and three-dimensional coupled problems. SIAM Journal on Numerical Analysis 2012; 50(1): 194-215.

21. D'Angelo C, Quarteroni A. On the coupling of 1D and 3D diffusion-reaction equations. Application to tissue perfusion problems. Math. Models Methods Appl. Sci. 2008; 18(8):1481-1504.

22. Secomb TW. Microvascular network structures. (Available from: www.physiology.arizona.edu/people/secomb/ network) [Accessed on November 2012].

23. Soltani M, Chen P. Numerical modeling of interstitial fluid flow coupled with blood flow through a remodeled solid tumor microvascular network. PLOS ONE 2013; 8(6):e67025. DOI: 10.1371/journal.pone.0067025.

24. Curry FE. Mechanics and thermodynamics of transcapillary exchange, chapter 8. In, Hand Book of Physiology. Am. Physiol. Soc., Bethesda. American Physiological Society: Bethesda, Maryland, 1984; 309-374.

25. Friedman MH. Principles and Models of Biological Transport. Springer: New York, 2008.

26. Jain RK, Tong RT, Munn LL. Effect of vascular normalization by antiangiogenic therapy on interstitial hypertension, peritumor edema, and lymphatic metastasis: Insights from a mathematical model. Cancer Research 2007; 67(6):2729-2735.

27. Perktold K, Prosi M, Zunino P. Mathematical models of mass transfer in the vascular walls. In Cardiovascular Mathematics, vol. 1, MS\&A. Model. Simul. Appl. Springer: Italia, Milan, 2009; 243-278.

28. Secomb TW, Hsu R, Braun RD, Ross JR, Gross JF, Dewhirst MW. Theoretical simulation of oxygen transport to tumors by three-dimensional networks of microvessels. Advances in Experimental Medicine and Biology 1998; 454:629-634.

29. Caputo M, Chiastra C, Cianciolo C, Cutr E, Dubini G, Gunn J, Keller B, Migliavacca F, Zunino P. Simulation of oxygen transfer in stented arteries and correlation with in-stent restenosis. International Journal for Numerical Methods in Biomedical Engineering 2013; 29(12):1373-1387.

30. Moore JA, Ethier CR. Oxygen mass transfer calculations in large arteries. Journal of Biomechanical Engineering 1997; 119(4):469-475.

31. Ganta S, Devalapally H, Shahiwala A, Amiji M. A review of stimuli-responsive nanocarriers for drug and gene delivery. Journal of Controlled Release 2008; 126(3):187-204.

32. Torchilin VP. Multifunctional nanocarriers. Advanced Drug Delivery Reviews 2012; 64(SUPPL.):302-315.

33. Frieboes HB, Mu M, Lowengrub J, Decuzzi P, Cristini V. A computational model for predicting nanoparticle accumulation in tumor vasculature. PLOS ONE 2013; 8(2):1-11.

34. Hossain SS, Hughes TJR, Decuzzi P. Vascular deposition patterns for nanoparticles in an inflamed patient-specific arterial tree. Biomechanics and Modeling in Mechanobiology 2014; 13(3):585-597.

35. Macheras P, Iliadis A. Modeling in Biopharmaceutics, Pharmacokinetics, and Pharmacodynamics, Interdisciplinary Applied Mathematics, vol. 30. Springer: New York, 2006. Homogeneous and heterogeneous approaches.

36. Barzegar-Jalali M, Adibkia K, Valizadeh H, Shadbad MRS, Nokhodchi A, Omidi Y, Mohammadi G, Nezhadi SH, Hasan M. Kinetic analysis of drug release from nanoparticles. Journal of Pharmacy and Pharmaceutical Sciences 2008; 11(1):167-177.

37. Mu L, Feng SS. A novel controlled release formulation for the anticancer drug paclitaxel (taxol): Plga nanoparticles containing vitamin e tpgs. Journal of Controlled Release 2003; 86(1):33-48. 
38. Renard Y, Pommier J. Getfem++: a generic finite element library in c++, version 4.2 (2012). (Available from: http://download.gna.org/getfem/html/homepage/) [Accessed on November 2012].

39. Quarteroni A, Sacco R, Saleri F. Numerical Mathematics, Texts in Applied Mathematics, vol. 37. Springer-Verlag: New York, 2000.

40. Farina A, Fasano A, Mizerski J. A new model for blood flow in fenestrated capillaries with application to ultrafiltration in kidney glomeruli. submitted.

41. Lei H, Fedosov DA, Caswell B, Karniadakis GE. Blood flow in small tubes: Quantifying the transition to the non-continuum regime. Journal of Fluid Mechanics 2013; 722:214-239. 